\title{
Pour une sociologie audiovisuelle du travail
}

Un entretien avec Monique Haicault, réalisé par Henri Eckert et Corine Eyraud

Monique Haicault, Henri Eckert et Corine Eyraud

\section{OpenEdition}

\section{Journals}

Édition électronique

URL : https://journals.openedition.org/itti/2099

DOI : $10.4000 /$ itti.2099

Éditeur

Université de Poitiers

\section{Référence électronique}

Monique Haicault, Henri Eckert et Corine Eyraud, «Pour une sociologie audiovisuelle du travail »,

Images du travail, travail des images [En ligne], 11 | 2021, mis en ligne le 01 septembre 2021, consulté le 18 février 2022. URL : http://journals.openedition.org/itti/2099 ; DOI : https://doi.org/10.4000/itti.2099

Ce document a été généré automatiquement le 18 février 2022.

Images du travail, travail des images 


\title{
Pour une sociologie audiovisuelle $\mathrm{du}$ travail
}

\author{
Un entretien avec Monique Haicault, réalisé par Henri Eckert et Corine \\ Eyraud
}

\author{
Monique Haicault, Henri Eckert et Corine Eyraud
}

Sociologue de formation et de pratique (thèse soutenue à l'Université Paris Sorbonne en 1969 sur la formation de jeunes techniciens, sous la direction de Jean Stoetzel), Monique Haicault passe quelques années au Brésil et dispense dès 1970, au département de sociologie de l'Université Toulouse 2, des enseignements nouveaux: Formation et emploi des Jeunes, Travail des femmes et, à partir de 1978 des enseignements complets appuyés sur des recherches: Sociologie et anthropologie des rapports sociaux de sexe, Image et communication sociale, Méthodologie de l'image, Temps sociaux. Elle communique à des colloques sur les thèmes de ses travaux: Le travail domestique, (Colloque de l'ACSES, «Le procès de travail», Paris VII Jussieu 1977), suivie d'une publication collective Travail domestique, famille du capitalisme (Critique de l'Économie politique, $\left.n^{\circ} 3,1978\right)$; Production et reproduction des rapports sociaux de sexe et de classes ( $X^{e}$ Congrès mondial de Sociologie, Mexico 1982) co-écrit avec Danièle Combes publié dans Le sexe du travail, PUG, 1984 (mis en ligne en 2021), aux Colloques de l'AISLF (Association Internationale des sociologues de langue française) en 1992, 1996, 2000, participe à la création, en 1992, du Comité $\mathrm{n}^{\circ} 4$, Sociologie des rapports sociaux de sexe.

Dès 1964 elle s'engage dans des équipes de chercheurs : Groupe des Sciences Sociales de la jeunesse du Centre d'Études Sociologiques (1964-1970), dans des groupes de recherche pluridisciplinaires qu'elle anime ou contribue à créer : le GRIEF, Groupe de Recherches Interdisciplinaires d'Études de Femmes Toulouse-Mirail (1979-1985); Femmes de valeur, travail sans prix, le travail à domicile. La dot et la valeur des femmes, Cahiers du GRIEF (Presses de l'Université du Mirail 1982); L'Atelier Production/ Reproduction APRE, IRESCO-CNRS (1982-1986), Un réseau de praticiens de l'image avec Anne Guillou, Réseau National pratiques audiovisuelles en Sciences sociales; des rencontres; trois Cahiers de réflexion pour une sociologie de l'image 1986-1993 (Cinémaction, éd. Charles Corlet 2013); enfin, depuis 1994, l'Équipe Femmes- 
Méditerranée-GeFeM-MMSH, Autour d'Agency, un nouveau paradigme pour les recherches de genre, Agency: un concept opératoire dans les études de genre (Rives Méditerranéennes, $n^{\circ} 42$ UMR TELEMME, 2012), Pour enrichir les recherches de genre, remarques tirées de l'expérience de séminaires à plusieurs voix, Genre, Révolution, Transgression (Presses de l'Université de Provence, « coll. Penser le Genre » 2015).

Elle publie, suite à un enseignement complet donné à l'Université d'Ottawa en 1999, L'expérience sociale du quotidien, corps, espace, temps (coll. "Théorie sociale » University Ottawa Press, 2000), qui rassemble plusieurs articles sur ses thèmes de travail.

Chercheuse associée au LEST UMR 7317 à partir de 1985 elle poursuit la réflexion : sur le travail domestique, Pertes des savoirs familiaux, nouvelle professionnalité du travail domestique (Recherches Féministes, vol. 7, Québec 1993); sur les temps sociaux, «Du temps du travail industriel à la pluralité des temps sociaux " (Où va le temps de travail ? Octares, Toulouse, 2000) ; sur le symbolique dans les rapports sociaux, Doxa et asymétrie sociale de sexe. Women's Studies, Manuel de ressources. (Point d'appui ULB Bruxelles 1994), "Le tiers inclus dans les rapports sociaux de sexe » avec Marie Blanche Tahon in Le genre de la catégorisation du sexe (Revue de sociologie et d'Anthropologie, $\mathrm{n}^{\circ} 5$, éd. L'Harmattan, 2001-2002); sur les modes d'organisation des pratiques sociales: les modes domestico-familiaux (articles et ouvrage sur La vie en deux et sur L'héritage du quotidien, avec Annie Fouquet); les modes de socialisation, La tertiarisation des activités parascolaires, Faire ou faire faire, famille et services (Presses Universitaires de Rennes, 1996); Socialisation domestique et norme temporelle. La famille une affaire politique ? Chronique féministe, Bruxelles (Université des femmes, 1995); les modes de mobilité dans l'espace urbain, La ville en mouvement, plurimobilité des retraités dans Marseille, Nature en ville (Les Annales de la recherche urbaine $n^{\circ} 74,1997$ ).

Elle a développé tout au long de son travail une réflexion sur l'image, le film de recherche et les apports de la méthodologie de l'image en sciences sociales dans différents séminaires et rencontres, La méthodologie de l'image peut-elle être utile à la recherche en Sciences Sociales ? 2010 (Archives ouvertes halshs -00498016). Elle réalise plusieurs films de recherche, trois vidéos et deux documentaires dans une série de 31 portraits de travailleurs à domicile coréalisée et coproduite (LEST Service de Recherches de l'INA), 1986, diffusée sur Arte 1989, Vivre et travailler chez soi, vidéo de 17 minutes puis, par une approche audiovisuelle de la place des femmes dans la politique de la ville, elle réalise trois vidéos d'une série Temps des Femmes, Temps des villes, Rennes, Marseille, Liège 2002-2006, diffusées dans le milieu de la recherche en France et à l'étranger, Marseille; Des femmes dans les lieux et les temps quotidiens (Cahiers du CERES Université de la Manouba Tunis 2003, Série Histoire). Au cours de sa carrière d'enseignante-chercheuse, elle a réalisé une douzaine de films de recherche sous forme d'articles-vidéo qui ont été déposés aux archives ouvertes HAL-SHS du CNRS, comme la plupart des articles, ouvrages, rapports de recherche et communications. 
Photo 1 : Monique Haicault en action (libre de droits).

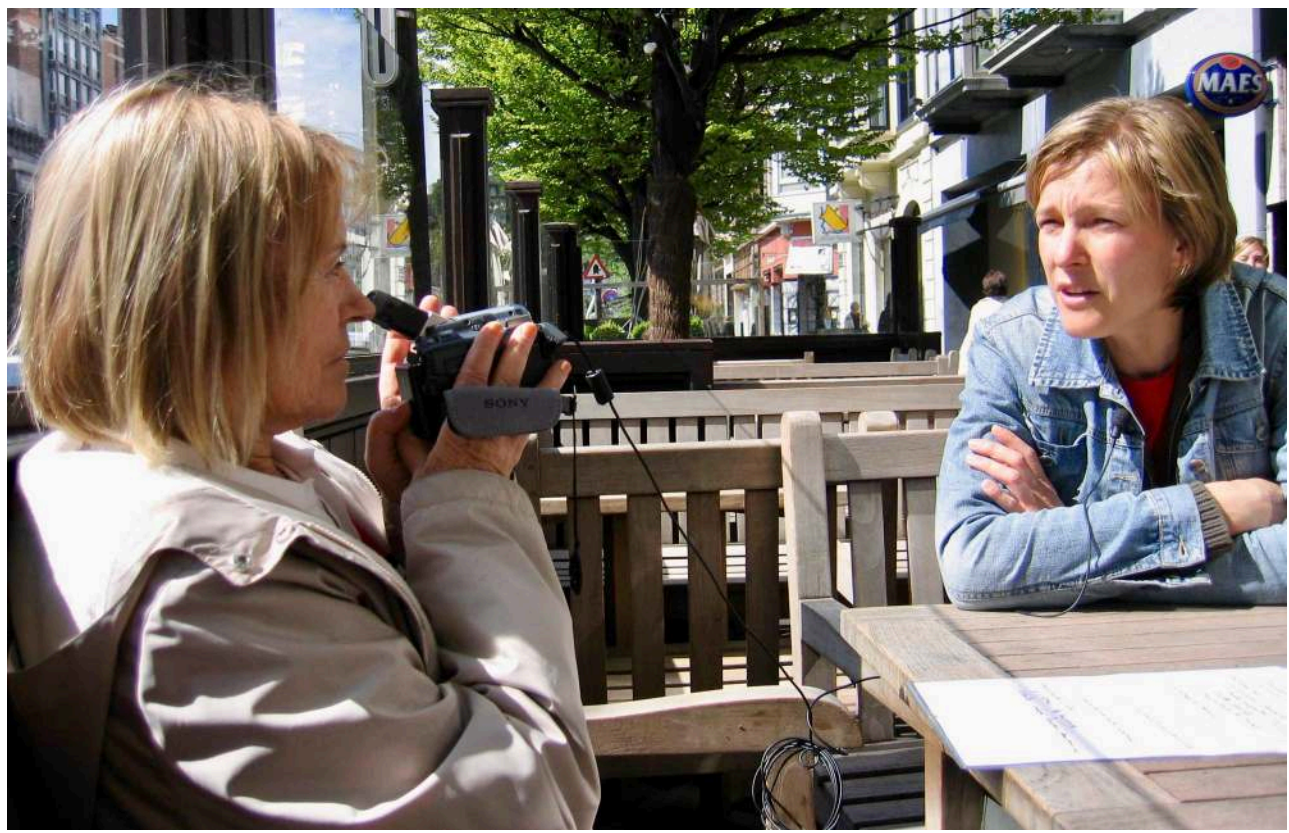

\section{Modalités de l'entretien}

Une série de questions, réunies dans trois points, avait été rédigée par les deux interviewers et transmise à Monique Haicault une semaine avant l'entretien. Monique Haicault a regroupé ces questions à sa manière, autour de trois thèmes, et nous a proposé d'entrée de jeu, dès le début de l'entretien, d'organiser celui-ci autour de ces trois points. La proposition nous convenait et l'entretien a, par conséquent, suivi l'ordre des questions telles qu'abordées ci-dessous par l'interviewée. Les interviewers se sont, par conséquent, contentés d'intervenir au fil de l'entretien, pour demander des précisions ou approfondir certains propos.

7 L'entretien n'a pu, du fait de la situation sanitaire fin mai/début juin, avoir lieu en présence des trois parties prenantes. Il a été réalisé «à distance ", selon l'expression désormais consacrée, le vendredi 4 juin 2021 à $14 \mathrm{~h}$. L'entretien s'est déroulé dans de bonnes conditions techniques, la spontanéité des échanges n'a pas été entravée. La qualité de l'enregistrement en a facilité la retranscription. La version définitive a été réalisée au terme de plusieurs allers-retours entre l'interviewée et ses interviewers.

Merci à Jean-Paul Géhin et Arnaud Mège pour leurs relectures.

\section{Entretien avec Monique Haicault}

Monique : Les questions que vous m'avez posées se regroupent autour de trois points. Le premier concerne mon parcours : comment j'en suis arrivée à faire de l'image et comment j'ai inséré cette pratique dans des cours. Le deuxième point renvoie à la méthodologie de l'image, comment je l'entends, personnellement. Le troisième point porte sur le programme d'une sociologie... Je n'aime pas trop ce mot visuelle », le son compte beaucoup, je pense qu'il faut garder "audiovisuelle», c'est important. Concernant mon parcours, j'ai encore trois points : l'enseignement, la recherche et la 
création de collectifs ou d'équipes. Concernant l'enseignement, j'ai commencé un cours sur le travail des femmes, des jeunes et des immigrés, dans le cadre de la sociologie du travail, dès 1970 à l'université de Toulouse. Dans le cadre de la sociologie du travail, j'ai été amenée à faire des films sur le travail domestique et sur le travail à domicile. Plus tard, entre 1982 et 1993, j'ai proposé un cours en master que j'avais intitulé «Image et communication sociale». La partie théorique sur l'image était prise en charge par Anne Sauvageot; mais elle, elle ne faisait pas de films. J'ai donc développé cette partie-là, un gros cours, accessible aussi en enseignement à distance. Je l'ai conservé dans mes archives, je vous le donnerai bien volontiers; il pourra encore servir à quelqu'un(e) ou à quelque chose! Ensuite, j'ai ouvert un atelier consacré à la formation et à l'analyse de l'image, toujours à Toulouse. C'était à l'époque de Raymond Ledrut, directeur du département, un sociologue, quelqu'un d'ouvert, qui a toujours accepté que je propose des enseignements totalement novateurs. À l'université, à cette époque-là, on pouvait créer des enseignements nouveaux, c'était un temps béni !

\section{Corine et Henri : [rires]}

Monique : J'ai proposé à chaque fois des cours innovants : sur le travail des femmes, ça n'avait pas encore été fait! Pour « Image et communication sociale, » il a fallu que je bâtisse complètement le cours et ça, c'était merveilleux! Puis «Sociologie des rapports sociaux de sexes » aussi, c'était complètement novateur. À chaque fois, dans le département, c'était accepté. Il y a eu aussi un enseignement à Ottawa, en 1999, où j'ai repris "Sociologie des rapports sociaux de sexes». J'ai amené les étudiants canadiens comme les étudiants à Toulouse, à utiliser l'approche audiovisuelle, je développerai ce point plus loin. Dans le cadre de cet enseignement, j'ai travaillé avec les étudiants sur les photos de famille. C'était magnifique! Mais je n'ai rien gardé de ça, hélas! Je ne vais pas trop développer mais enfin... on voyait très bien: les étudiants avaient apporté leurs photos de famille, c'étaient des moments extrêmement riches! On construisait ensemble les codes d'analyse, ça faisait réfléchir, par exemple comment se positionnent l'homme et la femme dans les photos de mariage. L'homme était toujours debout, posant sa main sur l'épaule de la femme assise, dans les photos d'avant la Deuxième Guerre mondiale, puis plus tard à côté l'un de l'autre. L'image parle toujours. On a travaillé aussi sur les photos de classe : on partait de l'idée que la photo de classe s'adresse aux parents, qu'elle dit quelque chose de l'école comme: «regardez comme on élève bien vos enfants!», entre autres! La photo est vraiment un outil de communication. On a travaillé aussi sur les rites de rencontre : les étudiants devaient, après s'être formés en atelier, filmer dans des gares, des lieux publics, des maisons de retraite, etc. Ils ont fait des choses magnifiques sur les rites d'interactions, les rites de rencontres, en référence à Goffman notamment ${ }^{1}$. On travaillait sur le corps, le langage du corps, l'hexis corporelle; du point de vue de la sémiologie tous les signes comptent. Les étudiants ont réalisé eux-mêmes des films. En 1982, ils ont travaillé sur une "sociologie des poubelles », le film s'appelait "Gaspiller c'est mal consommer ». On était en avance de 20,30, 40 ans sur l'extinction de la planète !

Corine et Henri : [rires]

Monique : Ils ont fait des merveilles de films, toujours à plusieurs mais pas plus de trois pour que chacun participe à tous les postes! Des films comme « La grammaire des crayons » : comment les inscriptions dans la ville, les tags, les graffitis signifient 
quelque chose... Ils ont produit de belles réalisations. Je ne développe pas trop ici tout ce que j'ai abordé dans mon cours ; je travaillais à la fois l'école de Palo Alto - « on ne peut pas ne pas communiquer ", de Gregory Bateson et les autres, E.-T. Hall et la proxémie, les corps dans l'espace - et tout ce qui relève du langage corporel, tout d'abord Marcel Mauss et les techniques du corps ${ }^{2}$ - plus tard Marcel Descamps notamment, et d'autres... ${ }^{3}$ - et aussi la théorie de la communication puisque l'intitulé était « Image et communication sociale ». Je partais aussi d'un questionnement sur ce qu'est la communication, les théories de la communication et, bien sûr, sur les médias du fait que le médium crée le message, comme l'affirmait le Canadien Mac Luhan. Etc. ! Donc, sur ce point-là, l'enseignement, avez-vous une autre question?

Henri : Une petite question, parce que vous avez prononcé le mot «sémiologie » et que, dans vos textes, vous faites souvent référence à Barthes...

\section{L'apport décisif de Roland Barthes}

Monique: Oui, bien sûr! Ça a été décisif pour moi, son article de 1964 sur la sémiologie dans la revue Communications ${ }^{4}$, numéro épuisé...

Corine : Oui...

Monique : «Éléments de sémiologie »... Il faut vraiment l'enseigner aux étudiants, c'était remarquable parce que Roland Barthes reprenait tous les concepts fondamentaux : "signifiant ", "signifié " bien sûr, mais aussi "connotation", " dénotation », etc. Tout ce qu'ont apporté les linguistes, Saussure dès 1915, affirmant l'arbitraire du signe comme " rapport entre un signifiant et un signifié », puis l'école de Prague avec Jakobson et les deux modes d'expression que sont la métaphore et la métonymie, qui peuvent s'appliquer parfaitement aux images. Ainsi peu à peu le passage de l'analogique au digital va faire disparaître le sujet et la complexité de la communication, celle du corps notamment que l'audiovisuel en quelque sorte peut restituer, une perte au profit de l'information binarisée. L'image est un signifiant et le travail sociologique consiste à décoder son signifié. Si on a un signifié que l'on veut transmettre par le langage de l'image, il faut choisir un bon signifiant. Il y avait donc tout ce travail réalisé par Barthes et ce n'est pas l'aspect structuraliste, son appartenance au courant structuraliste qui importe, mais plutôt ce qu'il apporte en tant que sémiologue et sociologue. Pas seulement dans Mythologies mais aussi ce qu'il a réalisé durant des années sur « Le texte et l'image », rassemblé en 1986 au Pavillon des Arts en une exposition mémorable conçue par Jérôme Serri. Barthes était un immense penseur, de ce point de vue là, un découvreur.

Henri : Est-ce que je peux me permettre de vous demander une petite précision ? Parce que, effectivement, signifiant, signifié, c'est intéressant, mais dans la langue, celle que nous parlons actuellement, on distingue facilement les signifiants. On peut aller assez facilement aux signifiés. Dans l'image, quelle est l'unité de base, quelles sont les unités de bases dans une image?

Monique : Il n'y a pas d'unité de base...

Henri : C'est ça...

Monique : Il y a une corrélation, articulée en permanence, entre signifiant et signifié. L'image ne parle pas vraiment d'elle-même, sauf à être construite, quand elle est intégrée dans un plan de recherche, qu'elle entre dans un processus de recherche: construction de l'objet, problématique et hypothèse. Donc elle est..., elle articule le 
signifiant et le signifié. Même les images, on va dire pauvres, qu'on a dans les reportages, il y a toujours un signifiant et un signifié. Sauf que le signifié renvoie alors à une signification émotionnelle. On choisit alors un signifiant qui renvoie à l'émotionnel : interviewer quelqu'un, un gros plan sur une flaque de sang, etc... Il y a toujours, à mon avis, un lien entre le signifiant et le signifié, un lien qui n'est pas toujours cohérent... Encore que ça serait tout un débat, il serait intéressant d'en parler. Mais pour un sociologue, il faut qu'il arrive à faire en sorte que le signifiant et le signifié soient cohérents, d'une certaine façon. Que l'image parle.

Henri : Oui, de faire parler l'image si je comprends bien?

Monique : Oui. Qu'on choisisse une image qui parle. Parce que faire parler une image, c'est tout un travail, qu'on faisait aussi dans le cours. On prenait des documentaires, on prenait un journal télévisé et on décodait: que dit l'image? Qu'est-ce qu'elle raconte ? Ça c'est très important mais tout ça, ça prend du temps... On verra à quel point, d'ailleurs je le dis tout de suite, c'est un des obstacles que rencontre la sociologie audiovisuelle : ça prend énormément de travail et énormément de temps. Et ça bouscule tous les présupposés, bien sûr. Il faut donc, sans arrêt, rechercher, travailler... Mais les étudiants étaient tous passionnés, ils choisissaient absolument tous ce cours-là quand ils avaient le choix. Ce n'était pas moi, personnellement ! C'était la richesse et l'intérêt pour eux du cours plutôt qu'un cours sur « Durkheim et le suicide », etc. !

Corine et Henri : [rires]

Monique : Il y a eu énormément d'apports, d'autres disciplines, de linguistes et même d'autres sociologues. Plus récemment de généticiens, des neurosciences, qui travaillent sur le cerveau, comment l'image s'inscrit dans le cerveau. Il y a toutes sortes d'apports nouveaux, dont je ne disposais pas à mon époque. Parce que j'ai quand même arrêté l'enseignement... Pas les films! Les films, j'ai continué après. Mais l'enseignement proprement dit...

Henri : Vous évoquez aussi Merleau-Ponty, un moment donné.

Monique : Oui ! Bien sûr, Merleau-Ponty ! Je l'ai eu comme professeur, parce que j'ai eu une formation de philosophe. J'ai même eu Bachelard, comme professeur ! Donc ça m'a énormément stimulée et rendue attentive à ce que Bachelard appelait les obstacles épistémologiques, c'est-à-dire tous les présupposés, les prénotions, les catégories normatives, etc. Un des apports de l'audiovisuel, lorsqu'on réfléchit sur l'image en lien avec une recherche, c'est qu'il nous oblige à changer nos catégories d'analyse et surtout nos catégories d'interprétation, ce qu'on enseigne très peu en méthodologie. Il y a les catégories de collecte de données, les catégories d'analyse, et après il y a l'interprétation. Et c'est là que la théorie intervient le plus, par les catégories d'interprétation. Là, on retrouve les travaux de Paul Ricœur, Le conflit des interprétations. Il y a toujours une herméneutique, une interprétation possible. Je trouve que l'image, si elle est telle que je l'imagine, et je pense que je ne suis pas la seule, elle est coûteuse en réflexion. Elle peut transformer les indicateurs et les catégories de lecture de la réalité sociale. Je n'emploie pas le mot « réel »... Comme le disait Edgar Morin, à propos du cinéma du réel, il faudrait se demander ce que c'est que le réel. Il n'y a pas de réel! Il y a des réalités sociales, construites lors de la construction de l'objet. Il y a des réalités sociales construites par les médias, sans arrêt. Les médias construisent des portions de réalité sociale, tout le temps les mêmes, avec les mêmes signifiants et les mêmes signifiés ! C'est devenu extrêmement 
normatif! Avec une réduction... affligeante ! Je me permets ce jugement de valeur. Affligeante parce qu'ils ont les moyens qu'on n'a pas... Que n'ont pas les chercheurs, on en parlera aussi !

Corine: Mais, avant que tu ne continues, tu nous as parlé du moment où tu avais commencé tes cours. Mais pas de ce qui t'a amenée à commencer... Ce qui t'a amenée à avoir l'idée et l'envie de faire des cours d'audiovisuel ?

\section{Une sociologie de terrain, qui s'intéresse aux situations et aux gens}

Monique : Voilà, c'est une conception de la sociologie qui m'y a amenée. C'est-à-dire que pour moi, la sociologie, c'est la sociologie de terrain, d'observation, la sociologie qui s'intéresse aux situations, qui s'intéresse aux gens. Donc une sociologie active, vivante et tout le temps en transformation. Ce qui ne veut pas dire qu'il n'y a pas de théorie. Au contraire! J'ai participé à la théorisation de beaucoup de concepts, notamment ceux de rapports sociaux de sexe, doxa de sexe et celui de charge mentale ${ }^{5}$. J'ai fait une communication à un colloque, via Zoom fin mai de cette année, sur la charge mentale et ce que j'ai travaillé depuis là-dessus. Donc, pour répondre à ta question, Corine, c'est une conception de la sociologie, qui consiste à aller voir. Aller voir, observer. Déjà à Toulouse, j'emmenais les étudiants voir les femmes en grève par exemple, dans le textile. Je n'avais pas encore fait de film mais j'avais fait un DESS, en 1959, je crois, avec Jean Stœtzel, où j'avais utilisé des images. Mon père avait des caméras, très tôt, on filmait! J'avais été initiée à une sorte d'usage du regard, comme... Comme les gens qui sont musiciens, qui ont souvent, une mère musicienne. Il y avait un milieu favorable, aussi une conception de la sociologie, et la pensée de Bachelard... On est plein de présupposés, donc il faut aller regarder, aller voir soi-même et ramener des données de première main. Et puis, il y a eu le colloque de l'ACSES, en 1977, l'Association pour la Critique des Sciences économiques et sociales. Le colloque portait sur le procès de travail, ça se passait à Jussieu. En petit groupe, Jacqueline Martin, des économistes de renom d'ailleurs, Annie Cot, Bruno Lautier, Dominique Fougeyrollas, avec aussi des chercheurs aujourd'hui disparus... On avait travaillé une année sur le travail domestique, est-ce que ça produit de la valeur, de la valeur d'échange, de la valeur d'usage, etc. ? Il y avait l'idée, assez répandue, que le travail domestique c'était la classe ouvrière, que les bourgeoises en étaient épargnées. Moi, ça m'a stimulée d'aller voir. Donc un été, l'été 1976, j’ai pris mes propres outils, puisque je n'avais jamais eu d'outils de la fac, aucun moyen... Mais ça c'est... Ce n'est pas un aparté, puisque ça explique pourquoi c'est si lent à progresser : parce que l'institution ne nous donne pas les moyens! On l'a vu dans le Réseau National crée en $1986^{6}$, les réticences académiques, on y reviendra peut-être tout à l'heure. Donc, je suis allée filmer, dans le grand ensemble du Mirail, les ouvrières de Motorola et...

\section{Corine : ...tu as été bien accueillie?}

Monique : Bien sûr que l'accueil était facile! Quand on explique aux gens ce qu'on veut regarder, ce qui nous intéresse, ils sont intéressés à coopérer, non pas pour se mettre en scène, c'est tout le contraire! Ils ne vont pas se mettre en scène, ils sont d'accord pour... Je rentrais chez eux et je les filmais en train d'accomplir les tâches domestiques, de parler de leur charge mentale, de choses comme ça. Et après, je suis allée dans la Sarthe et j'ai filmé ce qu'on peut appeler une bourgeoise, grande 
bourgeoise, femme de médecin, dans un lieu tout à fait idyllique... Toujours sur le travail domestique ${ }^{7}$.

Ce média ne peut être affiché ici. Veuillez vous reporter à l'édition en ligne http://

Henri : C'est elle qu'on voit à la fin de votre film sur le travail domestique?

Monique : Ah bon vous l'avez vu alors?

Henri : Oui ! Oui, ça m'a bien m'intéressé, oui [rires]

Monique : [rires] Ça me fait plaisir que vous l'ayez regardé ! Mais oui, c'est celle qu'on voit à la fin. Écoutez bon, vous voyez les difficultés ! C'est moi qui montais parce que je faisais tout moi-même, c'est moi qui montais, avec les outils que j'avais, et c'était très compliqué, parce qu'il a fallu réduire la durée... Mais j'aimais transmettre et montrer. Il y a donc des problèmes de désynchronisation de l'image et de la parole, surtout chez cette dame. Donc voilà, j'ai filmé, et, au colloque de l'ACSES, j'ai dit, à propos du travail domestique : "Non, ce travail-là, c'est pour toutes les femmes! » [rires]. Mais avec des moyens différents, qui sont des moyens de classe mais pas seulement. Qui tiennent à l'appartenance de classe mais aussi au lieu de vie, à l'inscription de l'habitat dans un espace urbain, périurbain, etc., toutes sortes de variables qui font que ce n'est pas seulement l'appartenance de classe, qui seule ne veut rien dire. On n'a pas les mêmes pratiques, les mêmes environnements et les mêmes contextes quand on est une bourgeoise, quand on est une femme de médecin dans la Sarthe, qu'on vit dans un lieu très choisi et quand on est une femme de médecin dans une banlieue parisienne. Bien évidemment ce ne sera pas la même pratique du travail domestique; mais bien évidemment que ce travail revient toujours aux femmes, une multiplicité de charges, qui relèvent du savoir-faire, de la gestion et du travail mental. J'ai répondu un peu à votre question?

\section{Corine et Henri : Oui !}

Monique : Alors, pour résumer ce qui m'a amenée à filmer, ça a été ce colloque. Après, j'ai filmé à peu près en même temps que j'enseignais. On a travaillé avec les étudiants, sur le travail à domicile, très développé alors. Il ne s'agissait pas de travail domestique, il s'agissait de travail salarié, pour le compte d'un employeur, effectué à domicile, dans différents secteurs de l'économie: le textile, la confection, les cravates, etc. Les femmes qui font des vestes de cuir... Vous les avez vues dans les portraits ${ }^{8}$ ?

Henri : Oui !

Monique : C'est énorme, la variété du travail à domicile! Et comme on travaillait avec les étudiants, ils découvraient que leur mère, leur tante, faisaient du travail à domicile, ils n'y avaient pas prêté attention. On a eu pléthore d'informations et moi, bien sûr, j'ai fait du terrain, régulièrement, dans le Sud-Ouest. J'allais voir ces femmes chez elles, j'ai intitulé ce premier film : «Des dames de qualité ». J'ai d'ailleurs écrit un texte là-dessus : " Femmes de valeur, travail sans prix », je crois que ça s'appelait. Bon, ces dames de qualité travaillaient chez elles : c'était encore la famille souche, plusieurs générations cohabitaient et les anciens travaillaient jusqu'au bout! Ils n'allaient pas dans des EHPAD! Ils travaillaient jusqu'au bout, les grands-mères surveillaient les enfants, s'occupaient des malades... Et dans ce film, « Les dames de 
qualité ", il y a une séquence où on voit très bien cette femme qui fait de la couture à domicile, qui va tous les jours à vélo chercher son travail et le ramène une fois fait. Et ça, l'image nous apporte ça: imaginez une question telle que «combien vous êtes payée? » ou « combien vous passez de temps?». Ces femmes étaient incapables de dire combien de temps elles y passaient ! Nous, on les interrogeait sur la journée de travail depuis le matin jusqu'au soir. Avec les étudiants, on voyait qu'il y avait des moments où elles tuilaient les deux activités, et j'en viens à dire très vite comment j'ai développé l'idée d'image-concept. Le poste de travail de la travailleuse à domicile est placé de telle manière qu'elle puisse voir l'enfant dans son berceau, ou dans la cuisine, la cocotte-minute qui souffle ou pas, ou l'enfant qui joue. Et je me suis dit: « voilà une image-concept. » Qu'est-ce que c'est une image concept ? Si on reprend la distinction entre signifiant/signifié, là, on a à la fois l'information du poste de travail et ce que j'appelle les rapports sociaux de sexe. Cette femme est en charge du travail domestique et du travail familial; il faut toujours ajouter familial à domestique ; aujourd'hui, la charge mentale tient beaucoup à la charge psychologique des enfants et des adultes, elle est familiale. Ainsi l'image-concept a un aspect matériel, signifiant : le poste de travail, la femme est assise, il y a un enfant qu'on voit, qui joue. Qu'est-ce que ça veut dire? Ça veut dire que cette femme a organisé son espace, qu'elle dispose d'un pouvoir d'agir sur son espace de manière à voir son enfant, pour le surveiller en même temps qu'elle coud. Voilà. On a un signifiant et un signifié qui sont cohérents mais qu'il faut décoder. En 1986 pour le service de recherche de l'INA, avec des réalisateurs professionnels, on a réalisé une série de 31 portraits de travailleurs et travailleuses à domicile, de laquelle j'ai tiré deux courts documentaires : «D'hier à aujourd'hui le travail à domicile » et «Vivre et travailler chez soi ", dans lesquels je propose plusieurs images-concepts`. À l'époque le matériel utilisé était très lourd, près de $10 \mathrm{~kg}$... Je me suis ruinée, en appareils audiovisuels ! Ils sont tous obsolètes, maintenant [rires] !

Corine et Henri : [rires]

Ce média ne peut être affiché ici. Veuillez vous reporter à l'édition en ligne http:// journals.openedition.org/itti/2099

\section{Saisir la complexité des situations et rendre compte des contextes}

Monique : Quand le caméscope est arrivé, ça a été la joie totale ! On pouvait filmer clandestinement! Le droit à l'image a freiné notre travail. Énormément. Je ne ferais plus les films que j'ai faits à l'époque, dans la rue, à Marseille... Certainement pas ! Quand je filme comment les femmes se déplacent, à quelle heure, dans quelle rue de Marseille, comment la ville est pratiquée par les femmes, je peux me mettre dans un café ou dans un coin de porte, j'ai mon petit caméscope et je filme. Bien sûr, quand on me demande " est-ce que vous lui avez demandé ? ", vous imaginez! Si on commence à demander aux gens "est-ce qu'on peut vous filmer ", d'abord ils vont dire non, parce qu'ils se disent "c'est mon intimité »; ensuite ils croiront qu'on veut faire de l'argent avec leur image! On ne peut pas demander! On ne peut pas prendre le risque de demander. Donc ça, c'est une vraie question, mais on en parlera peut-être après... on reprendra quand on parlera du programme de sociologie audiovisuelle. 


\section{Henri : D'accord!}

Monique : Revenons donc: qu'est-ce que m'apportaient ces images des années 1970 captées sur le terrain? D'abord, des données de première main. Ensuite, la complexité d'une situation, qui ne peut jamais être appréhendée par un questionnaire. Le contexte aussi, ce que je disais, sur un poste de travail. Quand j'ai filmé des hommes travaillant à domicile, jamais je n'ai vu d'enfant dans le champ. L'homme se met dans son garage, à l'abri des sollicitations domestiques et familiales ! C'est très intéressant. Ce n'est pas parce que l'homme est méchant ou mauvais, c'est parce que lui, il est à l'écart des sollicitations du travail domestique. Le contexte est une source importante pour comprendre une situation, le cadre social d'une situation, une séquence de mode de vie, une pratique urbaine de mobilité, il y a plein d'exemples... Une temporalité, mais ça c'est presque banal de le dire, un rythme, une durée... Quand on a créé le Réseau National à Nantes, on a vu - je ne dis pas ça pour critiquer -, que si on demande à un technicien de filmer un poste de travail, il va plutôt filmer la machine que la personne. On l'a vu d'ailleurs lors du colloque que tu as organisé, Corine.

Corine : Oui, tout à fait.

Monique : Plutôt filmer la machine, surtout si elle est un peu ostentatoire. Et moi je suis là, à me demander : " quand est-ce qu'il va filmer le travailleur? » [rires] Parce que c'est vraiment très important de voir la durée, le rythme, la fréquence, la pression temporelle. Je pense que pour tout travail répétitif, une question ne suffit pas... Ces opérateurs ne peuvent pas avoir la conscience de tout ce qu'ils vivent, sinon, ils éclateraient, ce serait le burnout. Donc ils s'économisent. Il y a une violence symbolique du questionnaire, c'est un aspect très important, d'ailleurs. La lenteur... la lenteur, le temps informe. Alors là, je fais référence à toute une série que vous avez peut-être vue, mais qui a été difficile à monter. C'était dans le cadre de «L'héritage du quotidien », une production de l'INSEE (Institut national de la statistique et des études économiques) et du PIRTTEM (Programme interdisciplinaire de recherche sur les technologies, le travail et les modes de vie, du CNRS), en 1992. J'ai travaillé avec Annie Fouquet, on a fait beaucoup d'entretiens, et j'ai filmé. J'ai filmé ce que j'appelle « Du réveil à la cloche »...

Corine: Oui, c'est vrai ! J'adore ! On en reparlait avant que tu n'arrives, j'adore, je suis allée les revoir ce matin! Quand tu as parlé de lenteur, j'ai revu ce petit paysan, le gosse qui part à vélo. Ah, c'est trop fort!

Monique : J'aime bien quand tu dis ça, parce qu'on se dit, quand on filme : « mais cet enfant-là, dans sa lenteur, il savoure ce que j'ai appelé la lenteur, le temps informe, le temps de la lenteur qui n'est pas le temps à rentabiliser ». J'ai travaillé sur ces catégories de temps, le temps de la lenteur que les enfants... Alors, selon les modes de socialisation, les familles des classes moyennes ont tendance à voler ce temps de la lenteur à l'enfant pour le rendre rentable dans la pratique scolaire. Tandis que ce petit enfant du Sud-Ouest, sur son vélo, il est heureux, il vit son enfance, le temps de la lenteur, ce que ne vivaient pas d'autres... Il y a ceux qui ne vivent rien du tout, ni la lenteur, ni rien... Ils sont là, devant la télé... Je pense que ça doit être effroyable maintenant, avec les nouveaux appareils. Le temps du rêve, le temps de la lenteur, du rien, de la réflexion... bon... ce temps-là. Et le temps du corps au travail, bien sûr, que je trouvais très important puisque, dans le cours que je faisais, j'utilisais aussi tout le travail qui a été fait sur le langage corporel, par l'école de Palo Alto, mais pas 
seulement. Sur l'hexis corporelle par Bourdieu... Tout le corps parle. Pas seulement comment il a vieilli, n'est-ce pas? [rires] Mais comment il se positionne ! Comment il s'habille! On voyait très bien chez les ouvrières à domicile dont je reparlerai tout à l'heure : comment cette femme coud des cravates pour Cardin. Parce qu'il ne s'agit pas du tout de bas de gamme, c'est du haut de gamme! Elle coud une cravate en trois minutes et demie, on a fait plusieurs prises, c'est exactement le même temps! Et son bras est devenu gros parce qu'il fait l'effort... Le corps se transforme. Danièle Linhart et d'autres l'ont dit, le corps se transforme au travail. Le corps parle le travail qu'il fait, non seulement l'âge... Le corps au travail, le corps dans l'espace urbain... Qu'estce qui fait une ville? C'est la ville-mouvement, les gens qui se déplacent, les corps qui se déplacent et pas seulement les bagnoles! Filmer comment les gens se déplacent... Quel droit de cité pour les femmes? J'ai bien vu à quel point dans Marseille, le droit de cité n'est pas pour toutes les femmes, c'est-à-dire le droit de cité seule, en tout lieu, en tout temps.

Henri : Pas qu'à Marseille [rires] !

Monique : Pas qu'à Marseille, mais j’ai filmé à Marseille! Quand elles se déplacent, elles sont à deux ou trois générations; très souvent il y a trois générations. Et là, comme disait Roland Barthes, «il faut savoir pour voir». Parce que faire un plan, comme ça, de femmes qui se déplacent... On dit : « tiens, il y a la fille, la petite-fille... » Eh bien là, ça dit qu'on se déplace en petit groupe familial ou amical. Mais des femmes seules, c'est à certaines heures et dans certaines rues. Au cours de la première rencontre audiovisuelle en 1986 à Nantes, on a découvert à quel point la question du rapport filmant/filmé, que vous avez soulevée, est importante, elle est en lien avec l'histoire sociale. On l'a évoqué tout à l'heure, c'était plus facile avant de filmer et d'avoir l'accord des gens. On obtient plus facilement une coopération sur des objets qui touchent les gens dans leur vie quotidienne que sur des objets du travail en entreprise. Parce que là, ça doit être très difficile. Je n'ai pas réussi à filmer en entreprise. Quand on a fait le film sur Motorola, un film que j'ai monté au moins dix fois, intitulé "Marie-Thérèse, la vie en deux", et... C'est une entreprise électronique, totalement secret défense. J'ai filmé une ouvrière, plusieurs ouvrières quand elles rentrent et quand elles sortent, le moment de l'entrée et de la sortie de l'usine, quand les cars de ramassage vont et viennent. C'est très beau ce carrousel, au début du film, une belle amorce de film, mais je n'ai pas pu franchir le portail de l'entreprise... J'ai filmé l'entreprise de l'extérieur, il était hors de question... On peut de moins en moins filmer en atelier...

Corine : Il y a aussi ce film où les parents te laissent seule avec les gosses nus, qui sont en train de s'habiller dans leur chambre! Quand tu parles de rapport filmant/filmé en lien avec I'histoire sociale, ce genre de scènes aujourd'hui n'est plus possible; regarder ces films aujourd'hui nous dit quelque chose des possibles et impossibles d'une époque... Filmer des gosses nus en train de s'habiller? En plus le père s'en va, tu es toute seule avec le petit garçon... ${ }^{10}$

Monique : Je suis seule!

Corine : T'es seule, avec le gamin!

Monique : Absolument !

Corine : $\mathrm{Nu}$, en train de s'habiller, dans son lit...

Monique: Je n'avais absolument pas pensé à ça mais tu as raison, bien sûr! C"était extraordinaire, parce que je leur avais demandé si je pouvais venir très tôt le matin... II y en a 
qui m'ont dit: «Mais venez dormir chez nous! » Je filme ce petit garçon, il n'est pas encore réveillé, il va faire pipi dans son pot, je continue de filmer, il descend etc. Mais c'est vrai, j'ai eu un accueil absolument... Et les gens avaient de l'intérêt, quoi ! C'est eux qui ont découvert que leurs pratiques avaient du sens et pouvaient intéresser les autres gens.

Ce média ne peut être affiché ici. Veuillez vous reporter à l'édition en ligne http:// journals.openedition.org/itti/2099

Henri : J'aimerais qu'on revienne sur ces petits films, que vous appelez des articles-vidéos ou des vidéos-articles...

Monique : Je voulais diffuser, parce que je trouve qu'on fait de la sociologie aussi pour informer les gens, pour casser un peu tous les présupposés véhiculés dans les médias... Les colloques, je n'avais jamais le temps... Se tenir au quart d'heure de parole quand il faut à la fois placer le film et la communication... C'est donc comme ça que m'est venue l'idée de faire un article-vidéo, avec des intertitres non pour dire « regardez ça »... mais un peu quand même: "Voilà ce qu'il faudrait que vous regardiez dans l'image!» [rires] Donner des contenus de recherche et amener à regarder, à s'interroger...

Henri : À former le regard...

Monique : À former le regard, c'est ça !

Henri : Oui ! On y reviendra à la fin de l'entretien! Oui, parce que je trouve ces articles-vidéos vraiment très intéressants... Est-ce que nous avons couvert tout le premier point?

\section{Création de collectifs et travail en réseau}

Monique : À peu près... Pour revenir au rapport filmant/filmé, il faut une coopération avec les gens qu'on interviewe et filme, qu'on ne rapte pas une image, surtout quand on est dans un milieu privé... À la rigueur, dans la ville, on rapte un peu... On prend une image mais chez les gens, il faut une coopération, il faut un accord, il faut établir une relation. Comme tu le disais, Corine, elle est aux prises avec tout ce qui se dit aujourd'hui sur le corps. Et puis, ce qui rend le rapport filmant/filmé très difficile, nous l'avons évoqué tout à l'heure, c'est le droit à l'image. Il faut tout le temps faire signer des chartes, c'est ce qu'on a fait pour la série de l'INA (Institut national de l'audiovisuel), d'ailleurs. J'avais écrit des choses sur le rapport filmant/filmé comme rapport d'imposition. On avait vu, dans ton colloque, qu'il y a une façon pour le sociologue de se présenter, qui peut amener le filmé à la profilmie ou à se mettre en scène lui-même... C'est un rapport très délicat à travailler, objet de beaux débats, de réflexion. Des collectifs de réflexion avaient été mis en place... des collectifs de chercheurs-praticiens. En 1973 à Toulouse, au département, on a ouvert des ateliers qui ont "boosté » la mise en place de la formation à l'audiovisuel au niveau de l'université, c'était intéressant... On a organisé, avec Marie-Thérèse Martelli, un colloque sur quelques jours, trois jours je crois, qui s'intitulait: "Une sociologie audiovisuelle est-elle possible?». Des anthropologues y ont participé, il y avait Colette Piault, Jeanne Labrune, il y avait Anne Pascal, Yvonne Mignot-Lefebvre, J'ai oublié les noms des gens autour de Rouch... Claudine de France... Et plus tard le Réseau... Dans le cadre du GRIEF (Groupe de recherche interdisciplinaire d'études des femmes), un collectif de chercheures pluridisciplinaires créé à Toulouse en 1979, on n'a pas mené une réflexion très poussée sur l'image. Mais on a fait un film sur 
l'argent, qu'on a présenté Marie-Laure Arripe, une économiste, et moi lors d'un colloque. Et un autre film sur les statues de la Vierge Marie sur les places des villages, intitulé : "Une femme exemplaire sur la place». J'ai filmé les Vierge Marie, qui apparaissent dans les villages du Sud-Ouest, sur un piédestal. Elles ont été érigées au milieu du XIXe siècle, au moment où la bourgeoisie mettait en place l'image de la bonne mère, bonne épouse. La référence à Marie était omniprésente. C'est un film que j'ai déposé aux archives ouvertes du CNRS. Mais celui sur l'argent, je n'en ai plus rien retrouvé. Je filmais en Super 8 à ce moment-là, il aurait donc fallu convertir les films en numérique, enfin bon... C'était intéressant parce que, sur les places de village $^{11}$, il y avait la Vierge Marie d'un côté et le Soldat de la guerre 14 de l'autre!

Henri : Le monument aux morts...

Monique: Voilà! Des choses qui parlent! [rires] J'avais interviewé les femmes, carrément: qu'est-ce que la Vierge Marie pour vous? Comment vous a-t-elle aidé dans votre vie? C'étaient des gens très catholiques, qui répondaient merveilleusement! Ce soutien que Marie apporte... L'une de ces femmes m'a dit: "Quand mon fils est mort, j'ai demandé à Marie, mais aide-moi, je ne comprends pas!» Elle n'est pas filmée à ce moment-là... Je ne l'ai pas filmée pendant qu'elle pleurait... Jamais ! Je déteste ça : jamais l'émotionnel ! Jamais l'intime ! Encore que tu me diras, [rires] l'enfant qui fait pipi dans son pot...

Corine : Les câlins du matin, c'est de l'intime... Mais de l'intime joyeux !

Photos 2 et 3 : deux publications du réseau.
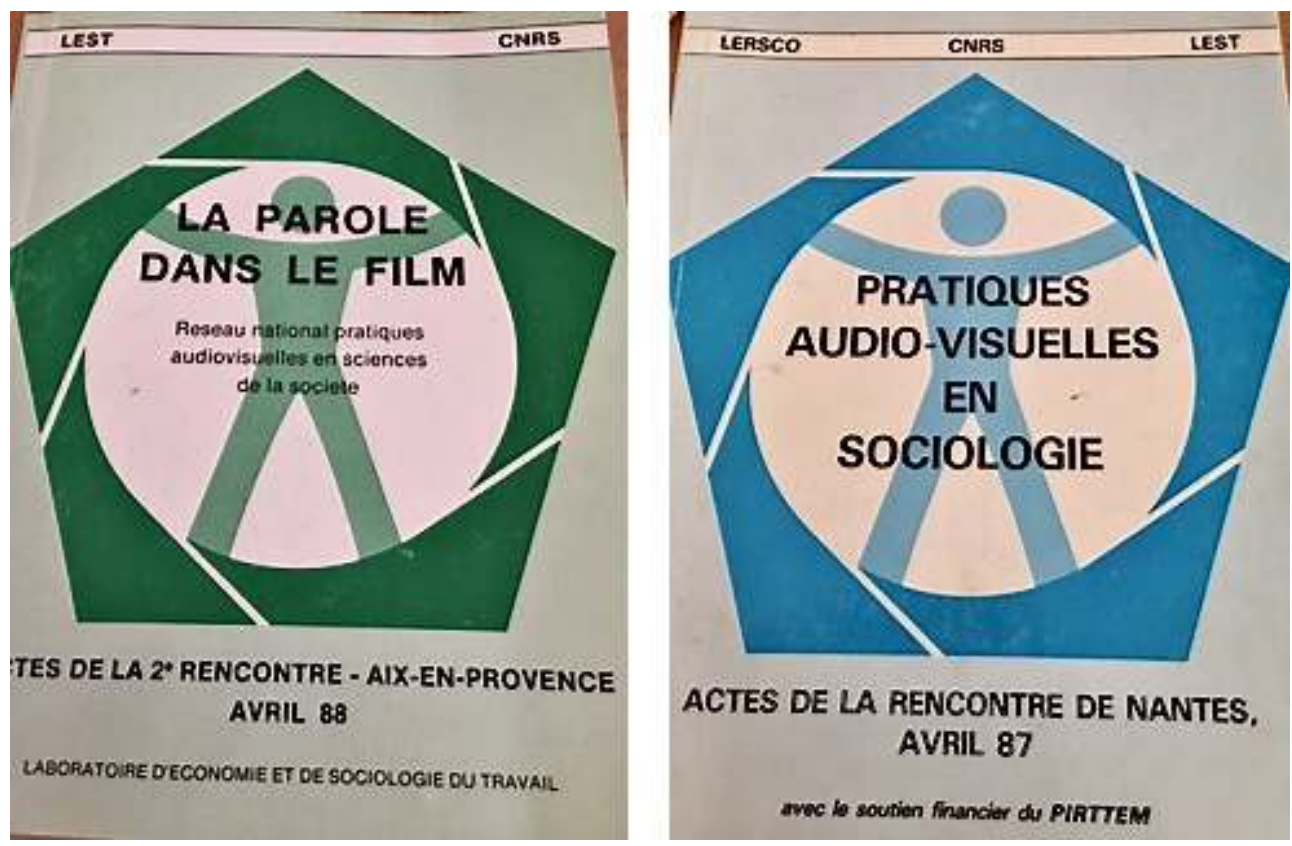

Monique : Revenons au Réseau National. Il a été créé avec Anne Guillou, qui utilisait aussi l'image. Tous les chercheurs du Réseau étaient des praticiens de l'image, à $99 \%$ des sociologues. Ils travaillaient avec leurs propres moyens, ils faisaient tout : image, montage, etc. Une vraie pratique. J'avais réussi à avoir un peu de crédit de Lucien Brahms, de la MIRE (Mission recherche au sein du ministère de la Santé et des Solidarités). Ça a été très long, très long d'avoir des sous, de faire accepter ce Réseau de praticiens. Il fallait qu'on se rencontre, payer les déplacements des gens, qu'un 
labo nous accueille: il fallait un minimum d'argent. Et on voulait qu'il y ait des Cahiers, que chacun fasse une communication, qu'elles soient rassemblées dans des Cahiers. Ces Cahiers sont sur le site du LEST (Laboratoire d'économie et de sociologie du travail, Aix-en-Provence), mais aussi dans les archives ouvertes depuis 2017. Il y a eu une première rencontre à Nantes... C'était Anne Guillou qui s'en était beaucoup occupée, moi un peu aussi. Puis à Aix, sur le thème «La parole dans le film ». Je n'ai pas retrouvé les Cahiers de la troisième rencontre, intitulée "La caméra sur le terrain ", qui s'est passée à Vaucresson. Pour Aix j'avais proposé que personne ne présente de films mais que chacun utilise un corpus de films qui circulaient pour travailler la parole dans ces films. Je les ai fait travailler comme des étudiants et ça a été absolument extraordinaire! Il y avait «La Rue du Moulin de la Pointe », un film de 1957... Ce qui est intéressant c'est qu'on a un commentaire off, qui présente l'analyse qu'avaient faite Jacques Krier et Jean-Claude Bergeret, d'après l'enquête de Paul-Henry Chombart de Lauwe sur la vie de la classe ouvrière à Paris... J'avais voulu qu'un documentariste de télévision chevronné assiste à la rencontre, c'était Hubert Knapp, qui est mort depuis, qui avait fait une analyse de la comédie documentaire « 5 Anglais pour Noël » et des stéréotypes dominants dans la fiction documentaire. Il y avait le film d'une Québécoise "What number?", sur l'introduction des nouvelles technologies. "Il était une fois la télé », un film de Marie-Claude Treilhou, qui était connue à l'époque dans le documentaire français. La « Rue du Moulin de la pointe »! Il ne faut pas oublier que, dans ces quartiers-là ${ }^{12}$, il n'y avait pas de salle de bain dans les appartements, il y avait un point d'eau dans la cour, il n'y avait pas de toilettes... Ce sont des films d'archives. "L'enfant aveugle", de Johan van der Keuken, extraordinaire film ! Je crois que Jean-Luc Lioult l'a utilisé dans son cours à la fac d'Aix. Un film de 1964, sur un adolescent aveugle, des heures d'enregistrements... C'était nouveau comme pour le film "The Store», un grand magasin à New York filmé par Frederik Wiseman... Ces films ont circulé et chacun devait faire une analyse de ce qu'il voyait dans le film. Ça a été la deuxième rencontre du Réseau. La troisième était intitulée "La caméra sur le terrain»: comment on vient sur le terrain, comment on utilise les outils... J'ai fait un compte-rendu de cette troisième rencontre du Réseau dans un numéro de Cinémaction de 2013...

Corine : « Sociologie de l'image, sociologie par l'image».

Monique: C'est ça, "Sociologie de l'image, sociologie par l'image», avec une interview d'Edgar Morin, précédée d'une introduction. L'interview portait sur «Chronique d'un été ». J'y ai écrit un article sur le Réseau...

Henri : J'aurais une question sur tout ça... J'ai l'impression qu'il y a eu comme une espèce d'effervescence à ce moment-là... Qu'ensuite, ça a... pas bloqué mais que ça a rencontré des réticences, des difficultés. J'ai l'impression que l'on vit encore aujourd'hui sur ces réticences ou ces difficultés, qui sont apparues après ce moment d'effervescence. Comment vous expliquez ça?

\section{Un film de recherche s'appuie sur une recherche}

Monique : Il faut peut-être situer tout ça dans la période historique... On est quand même dans la période riche en effervescences d'après 1968, qui va durer jusqu'en 1985-1990, pendant laquelle les gens innovent partout. Les gens inventent, innovent, s'emparent des appareils. Il n'y a pas du tout de différence de sexe. J'ai écrit quelque chose là-dessus, que les femmes se sont emparées de tous les outils de 
communication dès le début du cinéma. On n'en parle pas dans l'histoire officielle... Dans mon cours «Image et communication sociale ", j'avais montré que les femmes s'étaient emparées... Germaine Dulac et tant d'autres, qui se sont emparées des caméras au début du cinéma, comme nous, les femmes des années 70 , nous en sommes emparées. La vidéo légère! Il y avait une parole libre. On est entré, aujourd'hui, dans une période extrêmement coercitive, extrêmement normative, il faut le reconnaître et en prendre la mesure. Alors qu'à l'époque, il y avait une parole libre. On s'emparait des outils... Les chercheurs du Réseau faisaient entrer les outils dans leurs facs, utilisaient les bancs de montage qui s'y trouvaient. On négociait, on ne passait pas par toute une administration de... de machins très lourds. Il y avait une grande liberté de pensée, une grande effervescence dans plein de domaines... C'était l'époque du Nouveau Roman, par exemple, plein de choses comme ça... Mais surtout dans l'image, dans l'image! Les femmes et les hommes ont filmé. Et puis, il y en a qui ont pris ça en main, parce qu'il y a toujours un gros travail pour tenir ces collectifs, ces réseaux... Nous avons été des travailleuses! Je reviens sur le Réseau : on avait décidé, au début du Réseau, que ça circulerait, que chacun accueillerait une rencontre dans son laboratoire. À Nantes, ça a été au LERSCO (Laboratoire d'études et de recherches sociologiques sur la classe ouvrière), Anne Guillou, Pascal Guibert et Roger Cornu $^{13}$, qui avaient fait un premier film sur les petits-beurre LU. J'avais proposé d'organiser la deuxième rencontre à Aix-en-Provence, LEST (Laboratoire d'économie et de sociologie du travail). J'étais rentrée au LEST en 1985, j'ai eu l'accord des collègues ou des directeurs qui étaient des gens ouverts, Jean-Jacques Sylvestre, Alain d'Iribarne, etc., des gens ouverts à l'innovation. Après, ça a été à Vaucresson, au CRIV (Centre de recherche interdisciplinaire de Vaucresson) avec Michel Chauvière, un juriste, et Marie Cipriani-Crauste membre du Réseau. Il avait été question que d'autres prennent en charge... Bernard Ganne du Glysi à Lyon, Jean-Paul Terrenoire, Philippe Bonnin, Patrick Deshayes à l'IRESCO (Institut pour la recherche, le développement socioéconomique et la communication)... Mais bon... [rires]. Avec Marc-François Deligne Ils ont créé « Images et société ». C'est la vie des réseaux, c'est la vie des collectifs... C'est très intéressant sur le plan de la sociologie des mouvements sociaux, si on peut dire. Ça a été un mouvement social, vous avez raison de pointer ça, ça a été un mouvement social, le développement de l'image et l'effervescence des ciné-club, partout. On avait créé un ciné-club à Toulouse, à la fac et à la Maison des femmes au même moment, il y avait des ciné-clubs partout, tout le monde discutait de l'image... Et ça s'est arrêté... Terrenoire, donc, a fait «Images et société ». Ce n'était pas dans le même esprit: non pas montrer nos films mais réfléchir sur nos pratiques, faire avancer la réflexion, faire avancer les institutions surtout, faire reconnaître... Alors là je résume : faire reconnaître les films montés, pas des rushs mais des films montés: un document, un article vidéo, un article documentaire, comme un produit scientifique, un produit de recherche. Ça n'est jamais passé. À ma connaissance, ça n'est jamais passé comme l'est un article dans une revue, après avis d'un comité de lecture... Si le film avait été montré dans un colloque, après un débat, il aurait pu valoir dans la carrière de... Nous voulions aussi que l'institution nous donne des moyens. Non seulement qu'elle reconnaisse le produit mais aussi qu'elle donne des moyens. Que le CNRS donne des moyens... Il y en a eu un peu... [rires] pour " Images et société » à l'IRESCO. Moi, à Aix, je n'ai rien eu... Un contrat a permis de faire acheter un magnétoscope de lecture de films pour le LEST, des choses comme ça, mais je n'ai pas eu vraiment les moyens pour filmer. Bon, 
les directeurs, après l'effervescence, ont été plus soucieux de recherche classique... Il faut dire que la force de l'allant-de-soi, de la recherche "normale " comme disait Thomas Kuhn, elle est puissante en France! C'est le mort qui saisit le vif, comme disait Bourdieu! Donc euh... Je réponds un peu à votre question, mais sans faire de polémique... Il y a eu des choses, l'IMEREC (Institut méditerranéen de recherche et de création), à Marseille, a fait des choses intéressantes avec Jean-Pierre Olivier de Sardan et Pierre Jourdan, les noms me reviennent... Ils ont fait venir Jean Rouch, en 1993. On y a participé, j'y ai participé. J'avais racheté un caméscope, un HI8, ça valait la peau des fesses à cette époque-là [rires] de faire un film! J'avais présenté un film dans la continuité du Réseau. J'ai toujours défendu et je le défends encore, je ne dis pas que c'est la seule chose à faire mais je dis que c'est cardinal : un film de recherche s'appuie sur une recherche. Construction d'objet, problématique, hypothèses, catégories d'analyse, catégories d'interprétation, corpus théorique d'interprétation, c'est radical! On reviendra sur l'enseignement de la sociologue audiovisuelle... [à Corine] tu sais ce qui t'attend! Mais tu le fais déjà, certainement, ça paraît radical mais ce sont des garde-fous. Sinon on se retrouve avec des kilomètres d'images qui ne veulent rien dire... Des films sans réflexion, sans réfléchir à l'imposition des problématiques, comme disait Bourdieu!

Henri : Oui!

Monique : Et il y a eu aussi l'essoufflement, parce qu'il faut beaucoup... C'est une énergie... Un mouvement social, c'est une énergie qui s'inscrit dans le social. Comme le mouvement des Gilets Jaunes au début. Si on n'est pas capable de se dire : tiens, qu'est-ce que ça veut dire? Quelque chose qui naît, là ? Je suis allée sur les rondspoints, pour voir ce qui se passait, bon, c'est le minimum. On ne va pas dire qu'on va militer avec eux, mais c'est le travail du sociologue. Je vous laisse parler, maintenant.

Henri : Oui ! Votre rapport aux Gilets Jaunes nous intéresse aussi ! Mais auparavant, juste un petit détour par cette question de l'illégitimité de l'image ou du défaut de légitimité de l'image, une chose qui me travaille... J'ai poussé des étudiants à utiliser des images dans leur thèse, non pas des images mobiles, mais des images fixes. L'un a constitué tout un cahier d'images, qu'il a placé au début de sa thèse. J'avais trouvé ça très bien. Ce n'est pas passé complètement inaperçu mais presque, la soutenance a tourné autour du texte, du verbe, des mots... Et pratiquement rien sur les photos. J'aimerais donc que vous parliez un peu de cette question de la légitimité de l'image, l'image qui ne serait pas scientifique aux yeux de certains. Vous rapportez ça dans un texte : «l'image n'est pas scientifique » disentils. Pas vous mais ceux qui sont opposés à l'image...

Monique : L'image animée, c'est un divertissement... Ça a aussi à voir avec ce qu'a été la télévision. La télévision, au début, a produit des documentaires absolument extraordinaires, informatifs. Et petit à petit, c'est devenu du divertissement. Cette imposition du modèle télé compte aussi... Ça n'est peut-être pas très pertinent ce que je dis là, mais ça a joué... Sinon, c'est à l'intérieur de l'institution, la normativité de l'écrit. La normativité de l'écrit, encore maintenant... Vous venez de le dire... Je ne sais pas si c'étaient des images que votre étudiant a faites lui-même, si c'étaient des images qui illustrent... S'il a fait ses images lui-même, elles sont encore moins légitimes, aux yeux d'un jury que des images qui illustrent mais qui ont été faites par des professionnels...

Henri : C'était des images faites par lui.

Monique : La normativité... On ne prétend pas, quand on fait de l'image, qu'elle épuise la totalité de la signification. On ne dit pas ça, on nous renvoie ça, mais non! Elle 
apporte quelque chose, on y reviendra dans la méthodologie de l'image. L'image apporte quelque chose de différent. Le différent, ça gêne... L'image, elle n'a aucune prétention totalitaire sur la production de sens. Elle doit tout le temps lier, tout le temps énoncer sa limite.

Corine : Comme l'écrit devrait tout le temps énoncer sa limite, ce qu'il ne fait pas toujours...

Monique : Comme le questionnaire! Le questionnaire aussi devrait énoncer sa limite, dire les biais d'échantillon par exemple! On assiste à une inflation du chiffre... Le chiffre fait science, je ne sais plus qui a écrit ça... Mais le chiffre fait science, l'image n'est pas du chiffre. Le texte peut faire science dans les sciences molles que sont nos disciplines. Dans les sciences dures, il faut que ce soit du chiffre.

Henri : Le chiffre, c'est sérieux ! L'image ne serait pas sérieuse...

Ce média ne peut être affiché ici. Veuillez vous reporter à l'édition en ligne http://

Monique : Le chiffre domine aujourd'hui, dans notre société et je pense que ce n'est pas fini. $18 \%, 20 \%$ de victimes du covid, $15 \%$ dans tel département : on ne sait pas comment c'est calculé! On parlait du questionnaire: vous avez une différence de pourcentage: ça représente combien de personnes? Quelquefois très peu! On demande à l'image ce qu'on ne demande jamais au questionnaire et qu'on demande de moins en moins aux entretiens, pourvu qu'on fournisse trois pages d'entretien, même pas analysées. On n'entend plus la question sous-jacente. J'ai enseigné la méthodologie, c'est pour ça que je suis critique. Et que j'ai toujours été vigilante, y compris dans les entretiens. Je ne dis pas que je l'ai toujours fait parfaitement... C'est très difficile à mener, un bon entretien. On demande à l'image ce qu'on ne demande à aucune technique de collecte de données, pas même aux archives. Comment vous interprétez ces sources? "Je vois une femme qui regarde son enfant qui... $»^{14}$ : comment vous interprétez ça? Il faut introduire tout un corpus d'analyse, les rapports sociaux de sexe... Le positionnement dans l'espace n'est jamais le fait du hasard, sur un lieu de travail par exemple. Ailleurs, dans les autres lieux non plus! Il faut dire que les images... Il y a beaucoup d'images qui sont seulement bavardes, comme disait Godard ou je ne sais plus qui. Il faut se méfier des images bavardes ou des images stéréotypes. C'est coûteux de rendre l'image signifiante, de lui attribuer un signifié, un contenu conceptuel... C'est coûteux en réflexion et en cadrage... Je pense que dans la thèse de votre étudiant, ce serait intéressant... Est-ce qu'il avait analysé les images qu'il montrait?

Henri : Sans doute pas assez!

Monique : [rires]

Henri : Mais il avait fait lui-même la plupart de ses images, il avait produit ses images et il en faisait un usage complémentaire à son texte écrit, en essayant de rendre compte de ce qui se dit des rapports sociaux dans le groupe des travailleurs agricoles saisonniers... De ce qu'on peut lire des rapports sociaux à travers la lecture des images.

Monique : Alors c'est intéressant ! Je veux dire, c'est intéressant comme propos, de cerner les rapports sociaux dans une image, au sein d'un groupe. J'ai eu l'occasion de travailler avec et pour un étudiant afghan, il y a quelque temps... Il devait analyser un ouvrage d'un sociologue qui avait travaillé sur un chantier du bâtiment pendant un an ou deux, immergé dans le chantier. Il montre comment les Africains, les Noirs 
accomplissent les pires tâches ! Ça apporte beaucoup d'informations sur les rapports sociaux à l'intérieur d'un rapport social de dominés... C'est comme chez les femmes : il y a des rapports intrasexe. Les femmes, ce n'est pas une catégorie homogène! Les hommes non plus. La main-d'œuvre agricole non plus. Elle est travaillée par des rapports sociaux. Ce qu'il faut montrer, aussi c'est comment ça fonctionne...

Henri : Oui. Ce que je voulais dire surtout c'est qu'il y a comme pente «naturelle», comme vous le disiez tout à l'heure, à s'intéresser au texte et à trouver l'image plus anecdotique...

Monique : Oui, naturel au sens où c'est socialement construit.

Henri : Oui!

Monique : Ce n'est pas une nature!

Henri : Non, ce n'est pas une nature.

Ce média ne peut être affiché ici. Veuillez vous reporter à l'édition en ligne http:// journals.openedition.org/itti/2099

Monique : En 2005, avec le soutien du directeur, j'ai coorganisé une rencontre au LEST et à la MMSH (Maison méditerranéenne des sciences de l'homme), intitulée "Regards pluriels sur le monde du travail » ${ }^{15}$. Et j'ai demandé à des documentaristes et des chercheurs qui travaillaient sur les mêmes objets de recherche de comparer leurs pratiques de travail. Le film a été monté par un laboratoire parisien, ESCOM (Équipe sémiotique cognitive et nouveaux médias), il est répertorié aux archives. Ça a été un travail magnifique! On a beaucoup travaillé, on s'est réparti... Il fallait que je trouve un documentariste, un professionnel ayant travaillé sur un même objet, ça pouvait être une femme qui a travaillé sur la production mondialisée des blue-jeans et une chercheuse ou un chercheur qui a travaillé sur la question... Il s'agissait de répondre à des questions comme: quel regard portez-vous sur ce même objet? Quelles données avez-vous recueillies? Comment vous êtes-vous comporté sur le terrain ? Etc. La rencontre a duré deux jours. J'avais obtenu un soutien de la MIRE, enfin le LEST a bénéficié d'un soutien de la MIRE pour inviter documentaristes et chercheurs, des chercheurs du LEST surtout et des documentaristes nationaux pour la plupart, pas tous. Ça a été entièrement enregistré par le laboratoire ESCOM et après on a sélectionné les séquences pour les trois vidéos du montage... C'était intéressant parce qu'il y avait des débats, il fallait réfléchir sur l'image, voir comment un documentariste peut nous enrichir par sa technique, comment il est sur le terrain. Il y en a qui restent un an avant de commencer à filmer ! Ça a été une découverte, pour les chercheurs... qui quelquefois envoient un questionnaire par l'intermédiaire d'un syndicat! De l'autre côté, il y avait l'analyse des chercheurs... Les documentaristes ont été très intéressés. Deux milieux professionnels se sont rencontrés, qui travaillent sur des objets semblables et qui ont tout à gagner dans l'échange. Ça a fait évoluer la sociologie audiovisuelle!

Corine : Oui !

Monique : Ensuite, il y a eu ton colloque. Et le texte que j'ai écrit, dans lequel j'ai repris toute l'histoire, accompagnée d'une bonne bibliographie. Je n'ai pas présenté de film, on ne me l'avait pas demandé, d'ailleurs ! [rires] !

Corine: On a chacune son colloque ! [rires] Je l'avais organisé avec Guy Lambert, en $2007 \ldots$ 
Monique: Oui, 2007. Et puis en 2010, à l'École Normale Supérieure, Martine Sonnet a proposé une rencontre d'une journée sur le travail des femmes. Elle m'avait demandé si je pouvais venir... Elle voulait mon travail le plus ancien... J'ai remonté une séquence du film «Des Dames de qualité ». Ça a été la dernière fois que je suis intervenue dans des collectifs, des réflexions collectives. On est à la fin du parcours [rires]. II est 15 h 30 ! On continue?

Corine: Bien sûr! Mais tu as déjà dit plein de choses sur la méthodologie, que tu pourras reprendre...

\section{Filmer, une méthode heuristique}

Monique : Oui, et après il y aura le programme... Effectivement, on a déjà beaucoup parlé. On va donc aller assez vite. La méthodologie de l'image porte sur les usages, les intérêts et les limites de l'audiovisuel dans la recherche en sociologie. L'audiovisuel est un moyen, une méthode et un objet. C'est un moyen, parce qu'il apporte quelque chose de différent dans l'enquête, on l'a évoqué, sur les temporalités, sur le contexte, etc. Mais il est lourd, il est beaucoup plus lourd d'usage qu'un questionnaire ou un entretien. Il apporte quelque chose de différent, je ne reprends pas, sur le contexte, le corps, la temporalité, l'espace, sur la cohérence entre ces différents paramètres. C'est une méthode parce qu'il enrichit le corpus de données, encore faut-il les faire parler. C'est une méthode qui fait avancer le corpus de la recherche. Elle a une puissance heuristique, parce qu'elle apporte des informations sur des liens entre les variables, entre les dimensions, qui ne sont pas forcément prévues dans le questionnaire ou les entretiens. Elle permet de créer des nouveaux indicateurs pour la recherche, qui n'étaient pas forcément prévus. Par exemple, la notion de l'espace, de l'organisation de l'espace, un indicateur qu'on pourrait à la limite chiffrer: on est dans du périurbain ou dans une rue d'une grande ville, on est dans un espace rural... Elle permet d'inscrire dans l'espace, un espace toujours social... Dans un territoire, un territoire, toujours social. Elle permet de créer de nouveaux indicateurs : elle rend visible des données non verbales, des gestes, le corps, on l'a dit, le poste de travail, le rythme et elle transmet quelque chose d'important, le regard sociologique sur la question. Elle devrait avoir sa place à la télévision publique puisqu'elle forme le regard sociologique, un regard déformé par les médias et par le reportage qui cible toujours - c'est leur travail - mais qui cible juste le moment, délocalisé de tout contexte et surtout, qui vise l'émotionnel, pour que les gens fonctionnent à l'identification, à l'empathie. Or l'approche audiovisuelle ne fonctionne pas à l'empathie, elle doit mettre à distance l'empathie. Elle montre les liens entre variables et elle transmet un regard sociologique, elle permet de communiquer. L'image est un outil de communication. Dans la thèse évoquée tout à l'heure, les photos permettaient de communiquer quelque chose au jury, un contenu informationnel mais aussi communicationnel. Le documentaire sociologique doit avoir une place dans les politiques publiques. Construit, organisé autour d'une recherche, la méthodologie de l'image, c'est une certaine conception de la sociologie. Est-ce cela qui peut créer des freins ou des réticences? L'image contribue à l'intelligence du social, elle a cette prétention de donner plus de discernement, de contenu, on l'a dit. La méthodologie de l'image permet de revenir sur les contenus, ciblés, donc de multiplier les angles d'approche. Elle constitue en outre une mémoire. Par exemple: le travail domestique que j'avais filmé en 1976, une personne me l'a 
demandé il y a une dizaine d'années. Elle me disait : je fais la même chose mais tous les outils de cuisine, tous les outils domestiques, toute la technologie domestique ont changé. Comment le montrer mieux qu'à partir d'un film qui les a enregistrés à une certaine époque? Donc elle crée une mémoire, et ce que vous disiez sur l'enfant au réveil, c'est rappeler une mémoire, c'est une archive. Cette capacité d'archive, ça n'est pas rien.

Henri : Dans votre premier film, on voit très bien comment étaient les cuisines à l'époque. Les cuisines d'aujourd'hui ne ressemblent plus à celles-là... La cocotte-minute... Ça fait vraiment archive, ça m'a fait penser à la cuisine de mon enfance, quand j'y voyais ma mère faire la cuisine.

Monique: Oui c'est une archive. La méthodologie audiovisuelle archive aussi des portions de réalité sociale analysées, pas seulement du reportage, elle est plus riche qu'un simple reportage qui filme seulement un moment d'un événement. Là je filme une séquence de vie. On a tous les paramètres évoqués, matériels, non matériels... On voit la façon de faire, puisque vous évoquiez cette femme dans sa cuisine qui fait cuire des pommes de terre dans une cocotte-minute, je me souviens... On voit comment elle est habillée : une blouse, elle a mis une blouse entière pour faire sa cuisine. Comment elle se baisse, comment elle se met en scène. Pas pour le film, elle est l'opératrice dans sa cuisine. Il y a son hexis corporelle, elle se valorise, elle n'est pas une victime du travail domestique, elle en est fière. Il y a une dimension symbolique. Ses gestes, la temporalité de ses gestes, il y a une élégance dans ses gestes, tous ses gestes sont habités, elle n'est pas dans l'affolement, elle n'envoie pas les choses, voilà... Les autres aussi, que j'ai filmées. Il y a donc le symbolique, l'image de soi que l'on veut transmettre, la fierté du travail...

Corine : Et ça c'est des choses...

Monique : Comment les filmer?

Corine : J'allais te dire : ce sont des choses qu'on aurait beaucoup de mal à faire ressortir par l'entretien, tu ne saisirais pas forcément cette dignité, cette fierté du travail ou très difficilement.

Monique : La dignité ? C'est sûr que si on posait une question comme «est-ce que vous vous sentez digne? ? [rires]. Ce serait d'une violence...

Corine : Tout à fait.

Monique : ... alors que là, elle le vit, elle nous l'offre, c'est une relation.

\section{Pour un programme de sociologie audiovisuelle...}

Corine : Tu n'as pas parlé de l'objet... tu as dit que c'est un moyen, une méthode et un objet. Henri : Ah oui!

Corine : Ou alors l'objet tu l'as fait glisser dans la méthode...

Monique: C'est le produit monté, le produit d'une recherche. Et c'est là qu'on trouverait l'article vidéo... Un objet qui situe quelque chose à un moment d'une société, on l'a évoqué, un moment d'une société, d'une civilisation, un objet qui crée de l'archive, de la mémoire, c'est intéressant que l'on fasse des archives audiovisuelles quand même... 


\section{Henri : Absolument !}

Monique : Tout cela devrait être dans un projet de réseau, de praticiens ou d'une institution. Il n'y a pas que le CNRS, il y a les universités, peut-être qu'elles le font. Je ne suis plus dans le circuit depuis quelques années, peut-être que cela se fait maintenant. Dis-moi, Corine, toi qui sais tout aujourd'hui !

Corine : Eh bien j'ai un peu déconnecté en ce moment, je te raconterai pourquoi après, mais Henri le sait peut-être plus que moi... Je pense que nous vivons une phase régressive à beaucoup d'égards. II y avait quand même beaucoup plus d'ouverture jusqu'à la fin des années 1990 qu'il n'y en a ces années 2020, enfin surtout ces 5 ou 10 dernières années. Pour reprendre tes termes, la puissance de l'écrit au détriment de tout le reste, s'est renforcée... Je suis désolée, je ne suis pas très positive, nous vivons une période ambiguë avec un appel au développement de la "créativité », de l'«innovation » et des moyens alloués sur projets, mais en même temps une telle concurrence sur les postes que le critère des publications et du nombre de publications est de plus en plus puissant...

Monique: Alors, il y a plusieurs choses dans un programme de sociologie audiovisuelle, je ne sais pas s'il y a un ordre établi, mais bon... Il y aurait les grands domaines que sont la communication par l'image, toutes les théories de la communication, ce que j'avais entrepris dans mon cours sur la théorie de l'image. Un point serait l'histoire de la sociologie audiovisuelle en France, qu'on a brossé en partie, à partir de l'expérience que j'en ai eue, qu'il faudrait compléter, enrichir. Les liens avec l'anthropologie, comment l'anthropologie, telle que je l'ai connue, isole son objet, alors que la sociologie inclut l'objet dans une formation sociale en changement. Si on prend Jean Rouch par exemple, les Dogons, la colonisation, les femmes sont absentes, enfin bon, elles sont isolées, c'est son droit. Mais la sociologie, elle, ne peut pas isoler. Quelque part, elle est articulée, une histoire familiale singulière est liée à une histoire sociale générale, on le disait à propos d'une cocotte-minute [rires]. Si on voit une cocotte-minute toute seule... Mais si elle est incluse dans un travail sociologique, on a l'histoire sociale autour, on a l'histoire de la pénétration des technologies domestiques dans la famille, etc. Et l'évolution, comment la production capitaliste vise la famille et le domestique. Ce sont les thèmes de réflexion sur la charge mentale. Donc ses liens avec l'anthropologie comme dans les essais de création de réseau, ça fait partie de l'histoire de la sociologie audiovisuelle. Ça a continué, dans d'autres villes, il y a eu des créations de collectifs, je n'ai pas parlé de tout ce qui a été fait mais d'autres ont travaillé dans d'autres villes ${ }^{16}$. A mon avis, une histoire de la sociologie audiovisuelle en France doit parcourir tout ça, collecter toutes ces informations, et puis réfléchir et susciter peut-être des rencontres... Une réflexion sur les thématiques : quelles sont les principales thématiques? Alors toi tu dis souvent une sociologie audiovisuelle sur le travail plutôt, non?

Henri : Oui, enfin...

Corine : Rappelons que la revue s'appelle «Images du travail, travail des images »...

Monique: Pas travail domestique, travail à domicile, travail en entreprise, euh, télétravail, travail des soignants, toutes les sortes de travail...

Corine: La sociologie audiovisuelle sur le travail est la plus développée des sociologies audiovisuelles mais il y en a d'autres, donc d'autres champs possibles, encore moins explorés, il me semble.

Monique : Par exemple, tu penserais à quoi ? 


\section{Corine : Eh bien la socialisation familiale.}

Monique : Voilà, c'est un travail.

Corine : C'est un travail, on peut le dire aussi...

Monique : C'est une sorte de travail, un travail qui ne produit pas... Il faut se mettre dans l'idée que tout travail ne produit pas des biens marchands. Sinon on n'avancera pas, on restera dans l'atelier. J'ai écrit un article là-dessus, à propos du temps de travail ${ }^{17}$, la triple centralité : de l'atelier, le travailleur masculin, le salariat... Il faut qu'elle éclate cette thématique, au profit de tout ce qui est activité...

Corine : Oui...

Monique : ...qui produit des valeurs d'usage, des valeurs d'échange, du symbolique, qui produit du rêve.

Corine : Ces femmes qui se promènent dans la ville, on est dans l'activité ?

Monique : On n'est pas dans le travail.

Corine : D'accord.

Monique : Et donc, quels sont les objectifs des productions thématiques répertoriées : travailler sur les archives, l'histoire et les archives, la place du chercheur... On ne l'a pas beaucoup évoqué pour l'instant, j'aurais dû le mettre dans la méthodologie. Par rapport à un travail d'équipe, la question que pose le fait de déléguer la prise d'image à un technicien. Il y a un risque de perte, une perte du regard, il faut initier le technicien à ce qu'on veut filmer. C'est une relation qui n'est pas facile, parce que lui dit : « oui moi je filme ça, je ne peux pas cadrer là parce qu'il y aura trop de lumière, ton truc je ne peux pas le filmer... ", etc. Or c'est le regard du sociologue qui compte, le technicien doit absolument s'approprier le regard du sociologue qui, lui, doit être très précis. Il doit savoir, non pas ce qu'il va voir mais ce qu'il veut enregistrer, le cadrage, la séquence et son contenu, sinon c'est la richesse de l'information qu'il va risquer de perdre. La réflexion doit porter sur la place du chercheur dans l'équipe. Et puis le travail du montage... Ce n'est pas pour rien que les films grand spectacle sont montés des dizaines de fois ! La diffusion des produits dans le milieu des sciences de la société doit faire partie aussi du programme de sociologie audiovisuelle. On retrouve là toutes questions relevant de l'institution, de ses réticences à accepter l'audiovisuel. Les sciences de la société doivent évoluer sur ces questions-là. La diffusion dans le grand public, le rapport avec la télévision, quand est ce qu'on peut passer un film ? La série dont je n'ai pas parlé, qui a été faite en coproduction avec l'INA, a été diffusée sur la chaîne 7, tout l'été 1989, sur Arte ${ }^{18}$. L'histoire de la sociologie audiovisuelle comprend aussi ses liens avec la télévision grand public et, localement, avec les ciné-clubs, car les diffusions locales sont importantes. Et puis, dans l'histoire de la sociologie audiovisuelle en France, il faut évoquer les lieux de formation. Comment se forment les chercheurs? On a parlé de la formation du regard, de la formation à la lecture des images. Mais la formation technique... Il n'y a pas de formation, je ne sais pas, actuellement? Est-ce qu'il y a une formation à l'audiovisuel au CNRS, pour les chercheurs?

Henri: À Poitiers, il y a quelques possibilités de formation, il y a un peu de matériel disponible et des gens capables de nous apprendre à nous en servir...

Monique : Oui ! Mais c'est un peu bricolé ? Ce n'est pas... 
Henri : Oui, complètement bricolé...

Monique : ...ce n'est pas une formation institutionnalisée. L'image aurait moins ce statut d'illégitimité si les institutions, les universités et le CNRS considéraient qu'il faut une formation, une formation technique à l'utilisation de l'image. Aujourd'hui ça reste quand même sacrément rudimentaire. Avec les outils qu'on a, on peut faire du montage... Il fallait voir les bancs de montage à l'époque quand j'ai fait mes premiers films... c'était des bancs montages en U-matic, qui faisaient un à deux mètres de longueur, c'était énorme. Aujourd'hui on peut faire du montage sur son portable... Mais quand même, il faut une formation et là, si l'institution, CNRS ou université, considérait qu'un chercheur doit être formé à la technique de l'image, ou à l'image, ou à la lecture de l'image, ce serait déjà un pas en avant. Vous voyez, c'est quelque chose que je n'avais pas encore souligné assez à quel point la dimension formation tarde à rentrer dans la panoplie du chercheur, dans sa boîte à outils.

Henri : Parmi nous, dans le petit groupe qui s'occupe de la revue - nous sommes quatre - il y a une collègue, Laurence Elena, qui a pris sur elle de faire un master spécialisé dans l'usage des techniques audiovisuelles. Elle s'est formée en prenant sur son temps, à côté de son travail pour faire ce master.

Monique : On en est encore là en 2021, alors ! [S'adressant à Corine] Et toi, à la fac d'Aix?

Corine : On a toujours aussi peu de sociologues qui font de l'image, ils en utilisent mais n'en produisent pas. Quelques-uns, comme moi, n'en produisent pas directement ou n'en produisent pas seuls mais travaillent avec des cinéastes. A Aix, nous avons la chance d'avoir un département cinéma important avec des chercheurs en cinéma qui sont des réalisateurs, Guy Lambert par exemple, et qui ont une pensée sciences sociales forte.

Monique : Est-ce qu'il y a eu un poste, quelqu'un qui enseigne à la place de Jean-Luc Lioult, qui est à la retraite maintenant...

Corine : Oui.

\section{... qui met l'accent sur la pratique et la réflexion sur les pratiques}

Monique : C'est vraiment la pente de l'orthodoxie, de la rationalité. Et comme l'image paraît irrationnelle, on se replie sur la théorie de l'image. Il n'y a pas de prise en compte des pratiques. Bon... Mais la pente, c'est quand même celle-là et c'est assez désolant, on ne va pas vers le terrain, voir, observer, voir et analyser comment les gens sont abusés, même les plus intelligents, par les images de la télévision. Ça dit quelque chose de notre formation intellectuelle. Donc la formation! Alors, deuxième point très important dans un programme, qu'on a évoqué déjà mais qu'on reprend, c'est l'initiation à la lecture des images. En tant que sociologue, comment regarder, quoi regarder, être attentif à quoi? Je parle d'images faites. Que disent-elles, comment, quel langage utilisent-elles ? Celui des doxas de la télévision ? Parasitée par les stéréotypes ? etc. Il faut toujours avoir ça en tête, où sont les stéréotypes, les doxas? Se demander ce qu'il faut regarder. Donc regarder des documentaires, travailler la lecture de l'image. Ensuite la pratique, l'initiation à des techniques simples, sur de petits objets, en atelier. Ça, ce serait toujours dans le programme d'une sociologie audiovisuelle telle que je l'imagine. C'est dit rapidement, mais c'est lourd! Très lourd ! Et parler des obstacles, ça j'y reviens toujours. Les obstacles à la reconnaissance, de la place d'une sociologie audiovisuelle, dans le champ de la production de connaissance. Ça sera un des points de la conclusion. L'image dérange 
la science académique alors que le chiffre donne l'illusion de la preuve. Or l'image ne peut pas rentrer dans la formulation de la preuve, donc elle n'est pas scientifique. Elle est considérée comme non-scientifique parce qu'elle ne peut pas faire la preuve. La science, c'est celle qui produit la preuve de ce qui a été avancé, pour qui la reproduction de la même chose donne le même résultat, ce qui donne la preuve. L'image est hors de cette assertion qui fonctionne comme un dogme. Il faut réfléchir sur le dogme. Ça ne veut pas dire qu'il faut tout abandonner, mais qu'il faut y réfléchir car ce dogme, c'est une construction sociale. En tant que sociologue, on doit l'interroger. Un sociologue interroge tout, comme un philosophe... Elle est coûteuse, l'analyse des obstacles, coûteuse en temps, elle double le temps de la recherche, au minimum. Au minimum! Ce temps doublé, on le prend sur notre passion, notre passion à passer des nuits à monter... Mais on ne vit pas que de passion... Elle est en concurrence avec les médias grand public, ça c'est très important, dans les obstacles à sa légitimité. Oh mais un reportage, je vous envoie ça tout de suite là! Le reportage est de moins en moins informatif. Il faut faire travailler les étudiants là-dessus. Qu'est-ce qu'il y a dans un journal télévisé, dans un reportage? Qu'est-ce qu'on apprend? Quel est le rôle de l'émotion? Comment l'émotion masque-t-elle l'information? L'émotion délégitime l'information. Elle la masque, elle la fait quasiment passer pour inutile. L'image audiovisuelle, sociologique, exigeante, dérange le politique au final... parce qu'elle montre autre chose. C'est sans doute aussi un obstacle à la diffusion grand public, elle montre ce qu'on ne veut pas voir, elle montre que dans le télétravail, il y a une part du travail effectué qui ne rentre pas dans le salaire : la mise en place, l'organisation du travail. Elle montre tout ce qui est hors de ce qui est vraiment rétribué, ce qu'est vraiment la production de quelque chose. Or un travailleur n'est pas un robot. Un robot, d'accord, il travaillerait directement. Mais un travailleur, il arrive, il va au vestiaire, il met sa blouse, il a un problème d'enfant, il a mal à sa dent, etc... Il va produire ce jour-là d'une certaine façon, il est un être humain au travail, ce n'est pas un robot au travail. Il y a une sociologie du robot au travail et nous on voudrait une sociologie de l'homme au travail. Qu'il trouve du bien-être au travail, la femme avec sa cocotte-minute, etc... Donc ça dérange le politique.

Corine: Si je peux me permettre une chose Monique, tout ça, c'est vrai, si c'est bien fait, dans l'optique d'une sociologie audiovisuelle exigeante, je voudrais juste que tu le rajoutes. Parce qu'une sociologie audiovisuelle, ce n'est pas parce qu'elle est audiovisuelle qu'elle n'entre pas parfois dans les stéréotypes, dans la doxa. Parce qu'elle découpe elle aussi et peut ne pas permettre de visualiser ce qui est généralement invisibilisé...

Monique : Comme tu as raison, bien sûr, bien sûr... La sociologie audiovisuelle est une sociologie exigeante, bien sûr. Mais là on est dans un programme d'enseignement, donc on vise l'exigeant, l'excellence [rires]. Le dernier point, l'un des derniers dans le programme, concerne la manière d'écrire la sociologie avec des images et des sons. Comment construire, découper, organiser les images pour communiquer des connaissances. C'est autre chose que de donner à voir du social par l'image ou l'illustrer. C'est introduire un projet de connaissance, d'élévation de la conscience publique sur une question, c'est une école. C'est une école! Il faut travailler sur le langage, il n'est pas tout fait. Certainement que le langage qu'on utilisait il y a 30 ans ou 40 ans, les outils, les ressorts, le montage ne sont pas les mêmes; il y aura à travailler là-dessus. On est dans un langage vivant, toute langue est vivante! C'est un travail sur le langage, je le répète. La question que je me posais : je dis cela et je le 
montre, ou bien je montre l'image et je dis ce qu'elle dit. Vous voyez la différence ? Je reprends : je dis cela et je le montre. Je vais alors chercher une image qui illustre. C'est celle qui est le plus souvent admise d'ailleurs. Je dis cela : les ouvriers ont une baisse de productivité et je le montre. Mais il y a une autre manière : je montre une image et je dis - au sens chercher à lui faire dire ce qu'elle dit du point de vue du travailleur ou du rapport social dans lequel il se trouve - ce qu'elle dit. Il s'agit alors d'un projet éducatif. On fait travailler la réflexion sur une image. D'où cette question redoutable, qui ouvre tout un champ d'avenir pour les générations à venir, de traduire la sociologie en image. C'est-à-dire faire cohabiter et se mêler deux langages, le langage de la sociologie et le langage des images. C'est toute la difficulté : la cohérence entre le signifiant et le signifié, le signifiant étant du côté de ce qu'on voit, par exemple, et le signifié du côté du langage de la connaissance, par exemple, de ce qui veut être dit... Je montre une image construite par moi, sociologue, et je vous dis ce qu'elle dit : article vidéo !

\section{L’article vidéo}

Corine : C'est tout à fait ton article vidéo.

Ce média ne peut être affiché ici. Veuillez vous reporter à l'édition en ligne http:// journals.openedition.org/itti/2099

Monique: Merci Corine! [Rires] Alors... Respecter les codes déontologiques et notamment le droit aux images, de plus en plus redoutable et très compliqué ! Il y a eu un moment, au LEST, une bibliothécaire qui voulait que j'apporte un accord pour toutes les images que je mettais dans la médiathèque collective que j'installais au LEST, alors que je numérisais avec mes propres moyens tous mes documents vidéo et d'autres de chercheures du LEST souvent en cassette VHS, ou en Super 8. On ne pouvait pas se comprendre car chacune avait ses raisons. Que j'apporte l'accord de chaque personne figurant dans des documents faits 20 ou 30 ans auparavant, ici ou là et pour tout ! Ce n'était pas possible! Elle était dans sa logique, que je comprenais : il faut l'accord des gens filmés. Elle était dans sa logique. Il y a des logiques qui permettent peu de coopérer... Le droit aux images est vraiment coercitif, c'est un empêchement à faire des images même s'il est légitime...

Henri : ...jusqu'à un certain point, oui.

Monique : Mais en fin de compte, les gens sont filmés sans arrêt par les chaînes de télévision et ne donnent pas leur accord. Quand ils pleurent, alors là, ça va : c'est un accord. Ils sont mis en scène et c'est un accord. C'est méchant ce que je dis là mais... voilà ! Le droit aux images est venu, c'était légitime, tout droit arrive à un moment où un conflit dans la société demande à être résolu. Mais pour le sociologue de terrain, c'est un empêchement très puissant. La valeur heuristique de l'audiovisuel, il faut vraiment l'affirmer. C'est un peu l'objet de la sociologie: pousser à la réflexion. Parvenir à construire un programme qui pousse à la réflexion et enrichit la connaissance qu'une société a d'elle-même. C'est une discipline qui réfléchit sur ellemême, c'est la seule qui réfléchisse sur ce qu'elle produit. L'économie ne réfléchit pas, elle ne réfléchit pas sur ce qu'elle produit socialement, ce sont des sociologues qui vont produire la connaissance sur les effets de la mondialisation car la mondialisation elle-même ne réfléchit pas sur la pauvreté qu'elle produit, par exemple. La sociologie 
a cette ambition, c'est pourquoi elle n'existe pas dans les pays totalitaires, les premiers à aller en taule ce sont vous et moi, c'est immédiatement ceux qui produisent de la réflexion sur la société. C'est pourquoi, dans les pays totalitaires, c'est la sociologie d'enquête, qui produit du chiffre à l'aide de questionnaires - j'ai une ancienne étudiante qui avait travaillé là-dessus -, qui fonctionne. On passe par les syndicats, on soumet un questionnaire millimétré, on ne s'occupe pas de savoir... Les réponses, c'est oui ou non, aucun questionnement ou retour sur la question, sur pourquoi vous posez cette question-là. Qu'est-ce qu'il y a derrière votre question ? La sociologie qu'on prétend faire et qu'on veut faire, qui est une belle science, telle qu'on la pense, est dérangeante d'une certaine façon. Donc ça répond un peu à la question de l'effervescence des années 1970-1990. On voit aussi l'institution dans son immobilisme, qui ne va pas favoriser la formation, qui ne donne pas l'argent, qui refuse les films de recherche au profit des seuls textes... Sur tout ça, l'immobilisme des institutions, je ne sais pas comment faire bouger une institution : parce que tous les collectifs qu'on a créés, c'était aussi pour faire bouger l'institution!

Henri : Oui...

Monique : Voilà, écoutez, je vous remercie !

Henri : [rires] Non c'est à nous de vous remercier! Pas l'inverse! Mais je voudrais encore poser une simple question, en rapport avec le numéro dans lequel paraîtra notre entretien. C'est qu'on a lancé le numéro un peu de manière... Voilà, c'est de plus en plus difficile de filmer des gens ou de photographier des gens au travail. Moi je suis plus photo... On s'est dit qu'on allait faire un numéro dans lequel on photographierait ou filmerait le travail qu'on peut voir dans la rue... Dans la rue, il y a des chantiers, on refait une canalisation... II y a des gens qui font traverser les enfants à la sortie des écoles, il y a des laveurs de carreaux qui nettoient les vitrines des magasins...

Monique : Les vendeurs à la sauvette aussi.

Henri : Oui, tout à fait, voilà, les vendeurs à la sauvette...

Corine : Les livreurs Deliveroo ou autres qui ont envahi l'espace public...

Henri : Oui... Ma question porte sur ça...

Monique : Filmer dans la rue : on retrouve le droit à l'image! Il y a aussi la question de la distance. Si c'est à distance, il me semble qu'on ne va pas demander à un livreur: «est-ce que vous permettez que je vous filme?» On peut le filmer quand même, à une certaine distance. Il peut vous dire: «vous m'avez filmé dans mon intimité professionnelle », je ne sais pas. On est forcé de pirater là, non? Je ne vois pas, je ne vois pas comment s'en sortir autrement... Qu'est-ce qu'on disait, le laveur de carreaux ? Encore, oui, là on peut: je me verrais bien demander au laveur de carreaux : «j'ai envie de vous filmer pour montrer qu'il n'est pas si simple de laver les carreaux ». Parce que ce n'est pas simple de laver les carreaux ! Demander aussi : « combien vous en faites? ", « vous devez aller vite? ", voilà, et « vous permettez que je vous filme?». Toc, là c'est bon, là ça devrait marcher. Par contre, un chantier du BTP, alors là vous risquez de les avoir tout de suite sur le dos...

Henri : On peut faire des photos à la sauvette...

Corine: Une autre question, en lien avec ce que tu viens de dire à propos du laveur de carreaux. Ce que je trouve particulièrement important et précieux, dans ta manière de travailler, important pour ceux qui voudraient faire de l'audiovisuel ou les apprentis sociologues, c'est le respect des individus, Sinon tu n'aurais pas pu entrer dans les familles comme tu l'as fait... La manière que tu aurais d'aller voir le laveur de carreaux, le respect, mais le respect n'est pas le bon terme, plutôt une humanité qui suscite le respect mutuel 
avec les individus à qui tu parles, tu vois ? Je ne sais pas si je suis claire... Je voudrais bien que tu dises quelques mots là-dessus, en particulier parce que, dans ta partie méthodologie tu ne l'as pas dit... Ça renvoie également à une de tes règles déontologiques de restitution minimale du travail réalisé aux personnes que tu as filmées. Je trouve que c'est important d'en parler donc si tu pouvais bien nous en dire quelques petits mots...

Monique : Bon, rapidement. Merci d'avoir souligné ce que je n'ai pas assez souligné, le respect des individus. Le respect, c'est s'intéresser aux personnes qui font un travail. Le laveur de carreaux, c'est spontanément que j'ai dit: "votre travail est intéressant »; il faut utiliser cet outil-là, donner, comme tu viens de le dire, de la valeur à ce que font les gens. C'est plus que le respect, tu as raison de le souligner, mais il y a le respect et puis il y a quelque chose, je n'emploierai pas le mot empathie parce que ça n'aurait pas du tout sa place là mais... peut-être coprésence, je ne sais pas, quelque chose de cet ordre, au travail humain qui est là. De chercher à le comprendre et lui donner une place. Pas artificielle, on ne va pas dire que c'est intéressant d'être laveur de carreaux... Pas des trucs comme ça, tu vois, cette marge entre une fausse reconnaissance et une valorisation du travail lui-même, tel qu'il est, tel qu'il est fait. C'est-à-dire être proche et à distance en même temps. C'est une relation humaine à travailler hein, ça ne veut pas dire que je l'ai dans tous les cas [rires]. Ensuite le son, ah mais bien sûr, le son c'est tous les sons. Dans les documentaires, quand on met un son annexe, c'est épouvantable. Il y a eu une époque où on ajoutait aux documentaires une musique annexe. Il faut mettre le son du contexte. Le son parle, le son d'une machine, le bruit d'une machine; mais le son de la voix aussi, de la personne qui parle, etc., les sons ça fait partie de l'audiovisuel. Ils sont ceux d'un contexte, ils sont ceux d'une ville... Alors, au montage il faut les baisser parce que, sinon, ça peut devenir inaudible, mais la prise de son est importante. Le son, c'est une source d'information en soi aussi... Et du coup je dis sociologie audiovisuelle.

Corine : Quand on dit sociologie filmique il y a également le son et l'image mais tu préfères parler de sociologie audiovisuelle...

Monique: Audiovisuel ça renvoie aussi à des outils, tu vois, plus modestes. Non, audiovisuel, on est dans les outils audiovisuels, les générations de caméras, de caméscopes où on avait le son séparé. Avec les caméscopes récents, on a le son en même temps. On ne se rend pas compte de l'avantage que c'est d'avoir le son en même temps. Quelquefois il faut le couper. Jamais d'émotionnel, bien sûr ! On rejoint ce qu'on vient de dire à propos du respect et c'est la différence entre émotionnel et sensibilité. C'est très, très important, j'avais envie d'écrire quelque chose là-dessus ces derniers temps parce que je me disais : je ne supporte plus que les gens disent: «ah c'était plein d'émotions, j'ai eu plein d'émotions!» alors qu'il s'agit d'être sensible, d'éveiller nos sens, le toucher y compris, il y a du toucher quand on fait l'image, un toucher à distance. Le toucher à distance, l'ambiance, toucher un espace, les espaces habités ; il y a l'ouïe bien sûr, il y a la vue, et puis il y a les odeurs aussi, les odeurs, et puis les bruits de la ville enfin bon. Donc il faut éveiller nos sens, on pourrait dire : "vous avez aimé, c'était plein d'émotions ? ». Non, ça m'a éveillée... Il faudrait trouver... Le français est pauvre sur ce plan, c'est une langue trop dans la tête, qui est restée sur l'émotionnel et le mental. Je pense que rentrer dans un espace de travail, c'est déjà s'immerger dans le sensible. Non pas l'émotionnel, sur le mode : " oh, le pauvre! qu'est-ce qu'il travaille quand même », mais : " il y a une machine qui fait du bruit, et là il y a ça, et là il y a ça », " oh là là mais il y a des drôles d'odeurs 
là, ils utilisent un truc toxique! » Voilà, c'est les sens en éveil qui sont des capteurs d'informations, il faut retenir ça, alors que l'émotionnel n'est pas un capteur d'informations, il capte et diffuse les bas registres vibratoires de l'humain. Alors que la sensibilité, les sens sont des capteurs d'information. Donc voilà, merci Corine mais les questions restent entières, il faut travailler sur toutes ces belles questions-là.

\section{BIBLIOGRAPHIE}

Bachelard G. (1934) Le nouvel esprit scientifique, Paris, PUF, 181 p.

Barthes R. (1964) «Rhétorique de l'image », Communications, n² 4, 1964, pp. 40-51.

Descamps M. (1989), Le langage du corps et la communication corporelle, Paris PUF.

Eyraud C. \& Lambert G. (2009) Filmer le travail, films et travail ; Cinéma et sciences sociales, Aix-enProvence, Presses de l'Université de Provence, 216 p.

Goffman E. (1974) Les rites d'interaction, Paris, Minuit, 230 p.

Goffman E. (1979) La mise en scène de la vie quotidienne, Tome 2, Les relations en public, Paris, Minuit, $372 \mathrm{p}$.

Haicault M. (2021) La charge mentale, son émergence et ses transformations, un cadre conceptuel d'analyse. 2021. <halshs-03270240>

Mauss M. (1936), « Les techniques du corps ", Journal de psychologie, XXXII ? n 3-4 et dans (1950/2008) Sociologie et anthropologie, p. 363-386.

Ricœur P. (1969) Le conflit des interprétations, essai d'herméneutique, Paris, Le Seuil., 512 p.

Terssac G. de \& Tremblaye D. (dir.) (2000) Où va le temps de travail ?, Toulouse, Octares, 284 p.

\section{ANNEXES}

\section{Où trouver les films/vidéos sur le travail de Monique Haicault?}

(1976-2007) Le travail domestique - https://hal.archives-ouvertes.fr/medihal-01491680

(1986) Vivre et travailler chez soi - https://medihal.archives-ouvertes.fr/ medihal-01532390v1/

(1986) D'hier à aujourd'hui le travail à domicile - https://hal.archives-ouvertes.fr/ medihal-01532388

(1989) Jean-François, dans une ferme du pays toulousain - medihal-01561711v1

(1992) Mathieu, dans un loft sur le Vieux-Port à Marseille -. medihal-01561672v1 
(1993) Aurélie, en logement social à Marseille - medihal-01561689v1

(1993) Benjamin et Caroline, en pavillonnaire à Vitrolles - medihal-01561737v1

avec Fillon R. (2005). Regards pluriels sur le monde du travail (trois vidéos) :

- Dépossession du travail et de l'emploi - , https://medihal.archives-ouvertes.fr/ medihal-01564703/

- Fragmentation du travail et de l'emploi - , https://medihal.archives-ouvertes.fr/ medihal-01564754/

- Traitement de la main-d'œuvre - https://medihal.archives-ouvertes.fr/ medihal-01564774/

Le lecteur pourra trouver l'ensemble des productions de Monique Haicault disponibles aux adresses suivantes :

https://hal.archives-ouvertes.fr/search/index/?qa[authIdHal_s][]=monique-haicault ou

https://hal.archives-ouvertes.fr/medihal-01561803

\section{NOTES}

1. Goffman E. (1974), Les rites d'interaction et (1979) La mise en scène de la vie quotidienne, Tome 2, Les relations en public, Paris, Minuit.

2. Mauss M. (1936), «Les techniques du corps », Journal de psychologie, XXXII ? n³-4 et dans (1950/2008) Sociologie et anthropologie, pp. 363-386.

3. Descamps M. (1989), Le langage du corps et la communication corporelle, Paris, PUF.

4. Barthes R. (1964), « Rhétorique de l'image », Communications, n4, 1964, pp. 40-51.

5. Haicault M. (2021), La charge mentale, son émergence et ses transformations, un cadre conceptuel d'analyse. 2021. <halshs-03270240>.

6. Haicault M. (2013), «Réseau National Pratiques Audiovisuelles en sciences sociales (1986-1993) : des rencontres et trois cahiers de réflexions collectives pratiques et théoriques pour une sociologie par l'image ", Cinémaction, Sociologie de l'image, Sociologie par l'image $\mathrm{n}^{\circ}$ 147, $\mathrm{p}$. $169-175$ <halshs-01566784>.

7. Haicault M. (1976-2007). Le travail domestique, (1976-2007) Le travail domestique - https:// hal.archives-ouvertes.fr/medihal-01491680

8. Monique Haicault fait référence à une série qui a obtenu plusieurs prix internationaux et a été diffusée sur Arte à l'été 1989.

9. Dans cette série, Monique Haicault a été conseillère scientifique, elle a réalisé 3 des 31 portraits avec l'équipe technique et construit, avec une monteuse, les deux articles vidéo: (1986). Vivre et travailler chez soi, https://medihal.archives-ouvertes.fr/medihal-01532390v1/ et (1986) D'hier à aujourd'hui le travail à domicile - medihal-01532388v1

10. Haicault M. (1989) Jean-François, dans une ferme du pays toulousain medihal-01561711v1

et (1992) Mathieu, dans un loft sur le Vieux-Port à Marseille. medihal-01561672v1

et (1993) Aurélie, en logement social à Marseille. medihal-01561689v1

et (1993) Benjamin et Caroline, en pavillonnaire à Vitrolles. medihal-01561737v1 .

11. Dans le Sud-Ouest, sur les places de village, il y a souvent des statues de vierge sur piédestal, un poilu en face et plus loin le monument aux morts.

12. La rue du Moulin de la pointe est une rue du $13^{\mathrm{e}}$ arrondissement à Paris; le documentaire dont parle Monique Haicault avait marqué les esprits. 
13. Images du travail, travail des images a réalisé un entretien avec Roger Cornu, publié dans le numéro 5, 2018.

14. Cf. plus haut dans l'entretien, lorsque Monique Haicault parlait du travail à domicile et d'une femme qu'elle filmait pendant qu'elle travaillait tout en veillant sur son enfant.

15. Haicault M. \& Fillon R. (2005). Regards pluriels sur le monde du travail (trois vidéos)

- Dépossession du travail et de l'emploi, https://medihal.archives-ouvertes.fr/medihal-01564703/

- Fragmentation du travail et de l'emploi, https://medihal.archives-ouvertes.fr/ medihal-01564754/

- Traitement de la main d'œuvre, https://medihal.archives-ouvertes.fr/medihal-01564774/

16. Un début d'inventaire figure dans l'ouvrage du colloque organisé par Eyraud C. \& Lambert G. (2009), Filmer le travail, films et travail ; Cinéma et sciences sociales, Aix-en-Provence, Presses de l'Université de Provence.

17. Terssac G. de \& Tremblaye D. (dir.) (2000) Où va le temps de travail ?, Toulouse, Octares, 300 p.

18. Thierry Garrel alors directeur de la grille documentaire de la chaîne 7 Arte avait eu l'idée avec D. Guisard du Service de Recherche de l'INA de produire une série sur le travail à domicile et avait remarqué mon travail de sociologue sur la question.

\section{AUTEURS}

\section{MONIQUE HAICAULT}

Monique Haicault, docteure en sociologie (Sorbonne 1969), enseignante-chercheuse en sociologie à l'Université Toulouse 2 à partir de 1970, elle y crée des enseignements nouveaux : Formation et Emploi des Jeunes, Travail des femmes, puis à partir de 1978 : Sociologie et anthropologie des rapports sociaux de sexe, Image et Communication Sociale, Méthodologie de l'image, Temps sociaux. Chercheuse associée au LEST UMR 7317 à partir de 1985 elle poursuit la recherche et la réflexion sur trois thématiques : les temps sociaux, les rapports sociaux de sexe, la méthodologie de l'image. Elle contribue à créer plusieurs équipes de recherche, coresponsable de comités de recherche elle participe régulièrement à des colloques internationaux sur ces thématiques. Elle a publié de nombreux articles (notamment sur la charge mentale, les temps sociaux, le travail à domicile, les rapports sociaux de sexe), des ouvrages et des rapports de recherche, elle a réalisé et diffusé une douzaine de films de recherche sous forme d'articles-vidéo et une série Temps des Femmes, Temps des villes sur la place des femmes dans « La politique de la ville » dans trois vidéos, Rennes, Marseille, Liège 2002-2006.

\section{HENRI ECKERT}

Professeur émérite de sociologie à l'Université de Poitiers, membre de la direction collégiale d'Images du travail, travail des images. Il continue de mener des recherches au sein du Groupe de recherches sociologiques sur les sociétés contemporaines (GRESCO). Il s'intéresse plus particulièrement au devenir des classes populaires et à leur représentation. Il s'intéresse ainsi à l'analyse des images, leur production et leur utilisation dans le champ de la sociologie et des pratiques sociales en général. 


\section{CORINE EYRAUD}

Enseignante-chercheure en sociologie à l'Université d'Aix-Marseille, elle mène ses recherches au sein du Laboratoire d'Économie et de Sociologie du Travail (LEST). Ses travaux croisent les champs de la sociologie économique et de la gestion, de la sociologie de la quantification, de la sociologie de l'État et de l'enseignement supérieur.

Elle a travaillé avec des chercheurs en cinéma réalisateurs, en particulier Guy Lambert avec qui elle a organisé un colloque « Filmer le travail. Films et Travail » à Aix-en-Provence en novembre 2007. L'ouvrage (accompagné d'un DVD) Filmer le travail - Films et travail. Cinéma et Sciences sociales, co-dirigé avec Guy Lambert en est le produit. 